\title{
Propuesta de Conceptualización de Requisitos para Proyectos Software Basados en Formalismos de Ingeniería de Conocimiento
}

\author{
Lautaro Ferrer \\ Grupo de Investigación en Sistemas de Información (UNLa GISI) \\ Departamento de Desarrollo Productivo y Tecnológico \\ Universidad Nacional de Lanús \\ Remedios de Escalada, Buenos Aires, Argentina. \\ lautaroignacioferrer@hotmail.com
}

\begin{abstract}
Resumen-EI proceso de conceptualización de requisitos para Proyectos Software basados en formalismos de Ingeniería de Conocimiento presenta una serie de inconvenientes al identificarlos. En este artículo se presenta una propuesta de conceptualización de requisitos para Proyectos Software basados en formalismos de Ingeniería de Conocimiento que se estructura en dos fases: (a) Análisis Orientado al Problema: cuya finalidad es comprender el problema planteado por el usuario que concluye en la conceptualización del modelo de conocimiento para Proyectos Software, y (b) Análisis Orientado al Producto: cuya finalidad consiste en la obtención de los modelados que determinarán la manera de construir el sistema software en cuestión, dependiendo de lo analizado en la primera fase. Se proponen ocho tareas que articulan cada una de las técnicas que componen las fases del proceso propuesto.
\end{abstract}

Palabras Claves-Ingeniería de Conocimiento, Educción de requisitos, Conceptualización de Requisitos.

\section{INTRODUCCIÓN}

En esta sección se presenta el marco del artículo (sección I.A), se da una delimitación del problema (sección I.B), se plantean los elementos de la solución propuesta (sección I.C), y se da una visión general del proyecto (sección I.D).

\section{A. Marco Del Artículo}

La especificación de requisitos del software es una descripción completa del comportamiento del sistema software a desarrollar. Incluye la descripción de todas las interacciones que se prevén que los usuarios tendrán con el software. Las estrategias recomendadas para la especificación de los requisitos del software están descritas por la norma IEEE 830-1998. Este estándar describe las estructuras posibles, contenido deseable, y calidades de una especificación de requisitos del software. Los requisitos se dividen en tres: funcionales, no funcionales $\mathrm{y}$ organizacionales. Los requisitos "funcionales" son los que el usuario necesita que efectúe el software [1].

Por otro lado, los "no funcionales" son los "recursos" para que trabaje el sistema de información (redes, tecnología) e imponen restricciones al diseño o funcionamiento del sistema software. Y en el caso de los requisitos "organizacionales" podemos decir que son el marco contextual en el cual se implantará el sistema para conseguir un objetivo macro. Por otra parte la Ingeniería de Conocimiento es aquella disciplina moderna que forma parte de la Inteligencia Artificial, cuyo fin es el diseño y desarrollo de Sistemas Expertos (o Sistemas Basados en el Conocimiento). Para ello, intenta representar el conocimiento y razonamiento humanos en un determinado dominio, dentro de un sistema artificial. El trabajo de los ingenieros del conocimiento consiste en extraer el conocimiento de los expertos humanos en una determinada área, y en codificar dicho conocimiento de manera que pueda ser procesado por un sistema software. El ingeniero del conocimiento no es un experto en el campo que intenta modelar, mientras que el experto en el tema no tiene experiencia modelando su conocimiento de forma que pueda ser representado de forma genérica en un sistema software conocido como sistema basado en conocimiento. La educción de requisitos consiste en hallar e identificar los requisitos que debe satisfacer un determinado sistema de información. El proceso de conceptualización de requisitos para Proyectos Software basados en formalismos de ingeniería de conocimiento es una disciplina de los sistemas que facilita el problema a resolver por parte del profesional de ingeniería de software, antes de construir los modelos conceptuales que derivarán en el sistema a desarrollar. Realizar un buen entendimiento de lo solicitado por el usuario es el elemento clave para alcanzar el éxito del proyecto software.

\section{B. Delimitación Del Problema}

En el proceso de desarrollo de software y de acuerdo al enfoque tradicional, el ciclo de vida supone un modelo en el cual dicho producto evoluciona a través de una secuencia ordenada de transiciones de una fase a la siguiente conforme a una aproximación lineal o secuencial. Las fases del ciclo de vida del producto software son: requisito, diseño, implementación, pruebas y mantenimiento. La primera actividad correspondiente a la fase de "Requisitos" consiste en efectuar un entendimiento de las necesidades del solicitante. Es un problema abierto la poca uniformidad que se encuentran en las actividades de educción de requisitos. La necesidad de estructurar y categorizar la masa de información proveniente del proceso de educción a los efectos de facilitar la comprensión del problema manifestado por el usuario.

El problema abierto que se aborda en este artículo consiste en cómo se logra la representación de requisitos a partir del discurso del experto [2].

\section{Solución Propuesta}

La solución propuesta tiene dos fases: la primera basada en el análisis orientado al problema abordado, 
cuyo objetivo es la comprensión del problema planteado por el usuario, y la segunda basada en el análisis orientado al producto, cuyo objetivo consiste en la obtención de los modelados que determinarán la manera de construir el sistema software en cuestión.

\section{Visión General}

En la sección I ( Introducción), se presenta el marco del artículo, se da una delimitación del problema, se plantean los elementos de la solución propuesta, y se da una visión general del proyecto.

En la sección II (Estado de la Cuestión), se presentan los marcos teóricos del proceso de conceptualización de requisitos para esta línea de investigación.

En la sección III (Descripción del Problema), se identifica el problema de investigación, en el cual se resalta la dificultad encontrada para identificar los elementos para generar los diagramas de modelado, se define el problema abierto y se concluye con un sumario de investigación.

En la sección IV (Solución), se presenta un proceso de conceptualización de requisitos para proyectos software basados en formalismos de Ingeniería de Conocimiento, en el cual se abordan las cuestiones generales de mayor relevancia, se describe la estructura general de la propuesta. Se presenta una visión detallada del proceso de conceptualización de requisitos para Proyectos Software basados en formalismos de Ingeniería de Conocimiento, en la cual se describen los aspectos generales del proceso y el caso de estudio mediante el cual se ilustrará al mismo, se definen las fases que componen al proceso, se identifican las etapas que componen cada una de las fases, se señalan las técnicas y formalismos que se utilizan para el desarrollo de cada fase y se brinda un resumen del proceso.

En la sección V (Conclusiones), se presentan las aportaciones de este artículo y se destacan las futuras líneas de investigación que se consideran de interés en base al problema abierto que se presenta en este artículo.

\section{ESTADO DE LA CUESTIÓN}

En esta sección se presenta el estado de la cuestión sobre distintas teorías y técnicas que convergen con los objetivos del presente artículo. Se presenta la descripción del análisis y diseño orientado a objetos (sección II.A) junto a los diseños orientados a objetos para el artículo: Diagrama de Clases (sección II.A.1), Diagrama de Casos de Uso (sección II.A.2) y Escenario de Casos de Uso (sección II.A.3). Por otra parte, se presenta el marco teórico del modelado del conocimiento para el presente artículo (sección II.B), el cual se describen los conceptos: modelado del conocimiento (sección II.B.1), conocimientos fácticos (sección II.B.2), en la que se describe la tabla CAV (Concepto-Atributo-Valor) (sección II.B.2.a) y Diccionario (sección II.B.2.b). Por otra parte, se describen los conocimientos tácticos (sección II.B.3) en la que se menciona las tablas PER y los conocimientos estratégicos (sección II.B.4) en la que se describe el Diagrama Jerárquico de Tareas.

\section{A. Análisis y diseño orientado a objetos}

El análisis y diseño orientado a objetos es un enfoque de la ingeniería de software que modela un sistema como un grupo de objetos que interactúan entre sí. Representa un dominio absoluto en términos de conceptos, clasificados de acuerdo a su dependencia funcional. En este método de análisis y diseño se crea un conjunto de modelos utilizando una notación acordada como, por ejemplo, el lenguaje unificado de modelado (UML)

Ferrer, L.2016. Propuesta de Conceptualización de Requisitos para Proyectos Software Basados
[3]. El marco teórico del diseño orientado a objetos para el presente artículo, se estructura en base al marco teórico que se presenta en [3], identificando los siguientes conceptos: Diagrama de Clases (sección II.A.1), Diagrama de Casos de Uso (sección II.A.2) y Escenarios de Casos de Uso (sección II.A.3).

\section{1) Diagrama de Clases}

Un diagrama de clases es una presentación gráfica de la vista estática, que muestra una colección de elementos declarativos (estáticos) del modelo, como clases, tipos y sus contenidos y relaciones. Un diagrama de clases puede mostrar una vista de un paquete y contener símbolos de paquetes anidados. Un diagrama de clases contiene ciertos elementos materializados de comportamiento, como operaciones, pero cuya dinámica está representada en otros diagramas, como diagramas de estados o diagramas de colaboración. Este proceso y sus subproductos pueden ser visualizados gráficamente en la figura 1 .

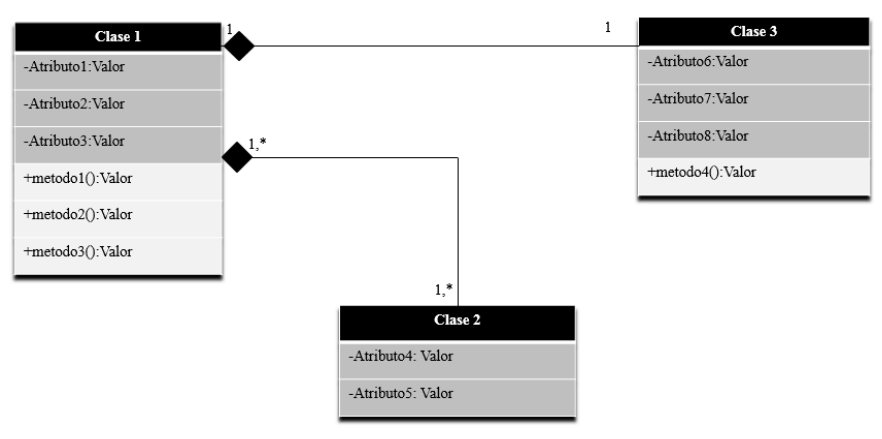

Fig. 1. Diagrama de Clases (DC)

\section{2) Diagrama de casos de uso}

Un diagrama de caso de uso es un grafo de actores, un conjunto de casos de uso encerrados por los límites de un sistema (un rectángulo), asociaciones entre los actores, los casos de uso y generalización entre los actores. Los diagramas de casos de uso muestran las relaciones existentes entre actores y casos de uso dentro de un sistema software a desarrollar. Este proceso y sus subproductos pueden ser visualizados gráficamente en la figura 2.

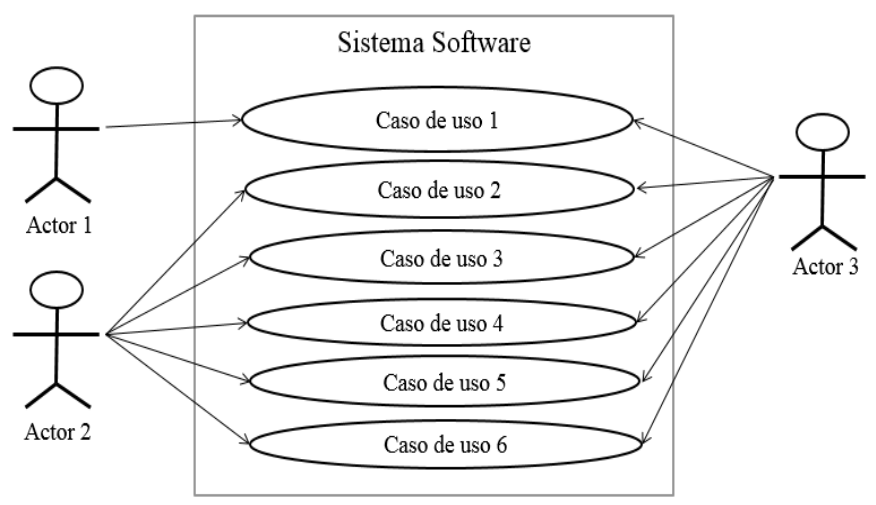

Fig. 2. Diagrama de Casos de Uso (DCU)

\section{3) Escenario de Caso de Uso}

Un caso de uso especifica la secuencia de acciones, incluyendo secuencias variantes y secuencias de error, que pueden ser efectuadas por un sistema, subsistema o clase por interacción con autores externos. Un escenario es una secuencia de acciones que ilustra un comportamiento y se puede utilizar para ilustrar la interacción o ejecución de una instancia de caso de uso. Este proceso y sus subproductos pueden ser visualizados gráficamente en la tabla I. 
TABLA I. ESCENARIOS DE CASO DE USO (ECU)

\begin{tabular}{|c|c|}
\hline Nombre del Caso de Uso: & Tarea/Subtarea n del DJT \\
\hline Área: & Nombre del Proyecto Software \\
\hline Actor: & Actor del Diagrama de Caso de Uso \\
\hline Descripcion: & Palabra del experto vinculada a la tarea n. \\
\hline Activar evento: & $\begin{array}{l}\text { Explicación de la palabra del experto concluyendo en un } \\
\text { evento activador ya sea un botón. }\end{array}$ \\
\hline Tipo de seíal: & Externa/Interna \\
\hline Pasos desemperiados & Información para los pasos \\
\hline 1) Paso 1 & Se describe el pase, 1 . \\
\hline 2) Paso 2 & Se describe el page, 2 . \\
\hline 3) Paso 3 & Se describe el page 3 . \\
\hline n) Paso $\mathrm{n}$ & Se describe el paso $\mathrm{n}$. \\
\hline Precondiciones: & $\begin{array}{c}\text { Se define la/s condición/es para desempeñar el caso de } \\
\text { uso en cuestión. }\end{array}$ \\
\hline Poscondiciones: & Objetivo conseguido al aplicar los pasos. \\
\hline Suposiciones: & Tiene un navegador, una clave, una contraseña, etc. \\
\hline Reunir reguerimientos: & Beneficios de aplicar este caso de uso. \\
\hline Aspertos sobresalientes: & Ej: ¿Se debe controlar el número de intentos? \\
\hline Pripridad: & Alta - Media - Baja \\
\hline Riesgo: & Alta - Media - Baja \\
\hline
\end{tabular}

\section{B. Marco teórico del modelado de conocimiento}

El marco teórico del modelado de conocimiento para el artículo, se estructura en base al marco teórico que se presenta en [4], identificando los siguientes conceptos: conocimientos fácticos (sección II.B.2), conocimientos tácticos (sección II.B.3), conocimientos estratégicos (sección II.B.4) y el resumen de artefactos mencionados para modelar conocimiento (sección II.B.5).

\section{1) Modelado del conocimiento.}

El Modelado del Conocimiento tiene como propósito dar forma automáticamente manipulable a los distintos tipos de conocimientos del dominio que maneja el experto. En esencia la mayoría de los dominios admiten que el conocimiento asociado se modele en términos de tres tipos de Conocimiento: Fácticos, Tácticos y Estratégicos.

\section{2) Conocimientos fácticos.}

Este tipo conocimiento es el relacionado con la descripción de los objetos conceptuales del universo de discurso del dominio de conocimiento sobre el que se pretende hacer un sistema experto. Este tipo de conocimiento se modela principalmente mediante dos técnicas: Tabla CAV (Concepto-Atributo-Valor) y Diccionario.

\section{a) Tabla CAV (Concepto-Atributo-Valor)}

La tabla CAV proporciona una lista de los conceptos que se manipulan en el dominio de conocimiento relacionado con la familia de problemas que resolverá el Sistema Experto a desarrollar.

Cada concepto quedará descripto en términos de los atributos que definen a cada concepto y de los valores que cada atributo puede tomar. Este proceso y sus subproductos pueden ser visualizados gráficamente en la figura 3.

\begin{tabular}{|c|c|c|}
\hline Conceptos & Atributos & Valores \\
\hline \multirow[t]{5}{*}{ Concepto 1} & \multirow[t]{3}{*}{ Atributo 1} & Valor 1 \\
\hline & & \begin{tabular}{|l|} 
Valor 2 \\
\end{tabular} \\
\hline & & Valor 3 \\
\hline & \multirow[t]{2}{*}{ Atributo 2} & Valor 1 \\
\hline & & Valor 2 \\
\hline \multirow[t]{7}{*}{ Concepto 2} & \multirow[t]{2}{*}{ Atributo 1} & Valor 1 \\
\hline & & \begin{tabular}{|l|} 
Valor 2 \\
\end{tabular} \\
\hline & \multirow[t]{5}{*}{\begin{tabular}{|l|} 
Atributo 2 \\
\end{tabular}} & \begin{tabular}{|l|} 
Valor 1 \\
\end{tabular} \\
\hline & & \begin{tabular}{|l|} 
Valor 2 \\
\end{tabular} \\
\hline & & \begin{tabular}{|l|} 
Valor 3 \\
\end{tabular} \\
\hline & & \begin{tabular}{|l|} 
Valor 4 \\
\end{tabular} \\
\hline & & Valor 5 \\
\hline
\end{tabular}

Fig. 3.Tabla CAV (Concepto-Atributo-Valor)

\section{b) Diccionario}

El diccionario proporciona una descripción de cada uno de los conceptos, atributos y valores que forman parte de la tabla CAV. Queda articulado mediante la cita del término y su definición ordenado lexicográficamente. Este proceso y sus subproductos pueden ser visualizados gráficamente en la tabla II.

TABLA II. DICCIONARIO

\begin{tabular}{|l|l|}
\hline Término & Definicion \\
\hline Concegto & ST que describe al concepto de la fila. \\
\hline Atributo & ST que describe al atributo de la fila. \\
\hline Valor & ST que describe al valor de la fils. \\
\hline Valor 2. & ST que describe al valor 2 de la fila. \\
\hline Valor a & ST que describe al valor n de la fila. \\
\hline
\end{tabular}

\section{3) Conocimientos tácticos}

Este tipo conocimiento es el que se refiera a las relaciones que vinculan los objetos conceptuales del universo de discurso del dominio de conocimiento sobre el que se pretende hacer un sistema experto. En esencia, la relación de más interés es la de causalidad entre conceptos, en particular, de qué modo se pueden inferir los valores de determinados atributos de determinados conceptos a partir de los valores que tienen otros atributos de otros conceptos (eventualmente los mismos). Este tipo de conocimiento se modela principalmente mediante el uso de reglas y se documenta mediante el uso de Tablas PER (Palabras del Experto-Regla). En una tabla PER se plantea el cuerpo del conocimiento (que contiene las relaciones de causalidad explícitas o implícitas identificadas) y la regla o reglas que lo modelan. Este proceso y sus subproductos pueden ser visualizados gráficamente en la tabla III.

TABLA III. TABLA PALABRAS EXPERTO REGLA VÍNCULO (PER)

\begin{tabular}{|l|l|}
\hline Identificador de la regla & \multicolumn{1}{|c|}{ Nombre de la regla } \\
\hline Palabras del experto & "...ccion"... \\
\hline \multirow{3}{*}{ Regla } & $\begin{array}{c}\text { SI Condicionl } \\
\text { Y Condicion2 } \\
\text { ENTONCES } \\
\text { Valorl - Valor1 + Valor2 }\end{array}$ \\
\hline
\end{tabular}

\section{4) Conocimientos estratégicos}

Este tipo conocimiento es el relacionado con la manera en que las distintas partes del dominio de conocimiento sobre el que se pretende hacer un sistema experto, son aplicadas para la resolución de una tarea. Con distintos niveles de granularidad, describe: (a) que es lo que hay que hacer, (b) bajo qué condiciones puede hacerse y (c) que post condiciones resultaran de lo que se haga. Este tipo de conocimiento se modela principalmente mediante la técnica Diagrama Jerárquico de Tareas [4]. En el Diagrama Jerárquico de tareas quedan especificados: (a) que subtarea compone cada tarea $\mathrm{y}$ (b) que información recibe $\mathrm{y}$ entrega cada tarea/subtarea. Este proceso y sus subproductos pueden ser visualizados gráficamente en la figura 4 .

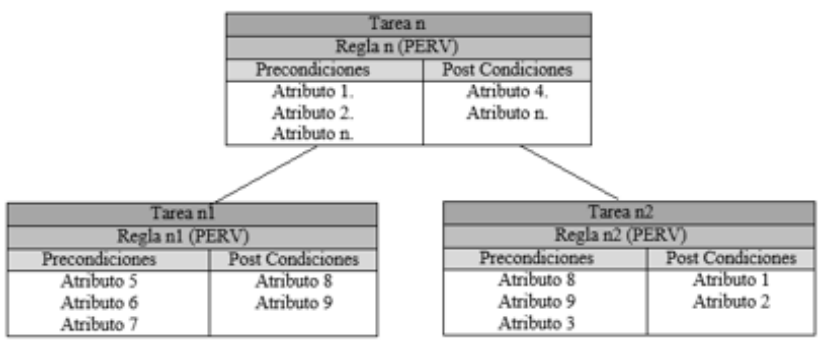

Fig. 4. Diagrama Jerárquico de Tareas.

Ferrer, L.2016. Propuesta de Conceptualización de Requisitos para Proyectos Software Basados en Formalismos de Ingeniería de Conocimiento Revista Latinoamericana de Ingeniería de Software, 4(5): 216-230, ISSN 2314-2642 


\section{DESCRIPCIÓN DEL PROBLEMA}

En esta sección se presenta el problema de investigación a analizar en el artículo, comenzando por la importancia que tiene la actividad de requisitos para la comprensión del problema de investigación (sección III.A), se caracteriza el problema abierto (sección III.B) y se concluye con un sumario de investigación (sección III.C).

\section{A. La actividad de requisitos en la compresión del problema.}

En el proceso de desarrollo de software y de acuerdo al enfoque tradicional, el ciclo de vida supone un modelo en el cuál dicho producto evoluciona a través de una secuencia ordenada de transiciones de una fase a la siguiente conforme a una aproximación lineal o secuencial. En este sentido, el ciclo de vida del producto software se ha estructurado en las siguientes fases: Requisitos, Diseño, Implementación, Pruebas y Mantenimiento [5].

La primera actividad correspondiente a la fase de "Requisitos" es muy importante en el desarrollo de un software ya que consiste en efectuar un entendimiento de las necesidades del solicitante. Frente a la poca uniformidad que se encuentra en las actividades de educción de requisitos [6], en este presente artículo se pretende sistematizar el procedimiento siguiendo lineamientos específicos. Realizar un entendimiento del problema permite comprender la necesidad del usuario y como ésta necesidad debe ser resuelta o satisfecha. El Ingeniero de Requisitos (IR) debe, mediante indagaciones, relevar temas de interés para tener un entendimiento del tema abordado.

\section{B. Problema abierto}

Existe la necesidad de estructurar y categorizar la masa de información proveniente del proceso de educción de requisitos a los efectos de facilitar la comprensión del problema manifestado por el usuario [5] [6] [7]. El problema abierto que se aborda en este artículo consiste en la existencia de una "brecha conceptual" en la transición del proceso de conceptualización de requisitos entre el Discurso del Usuario y los Requisitos. Este concepto se ilustra en la figura 5.

Se manifiesta la necesidad de conceptualizar los requisitos manifestados por el usuario en su discurso antes de crear los modelos conceptuales para el proyecto software en cuestión. El objetivo es reducir la complejidad $\mathrm{y}$ favorecer la comprensión de la problemática abordada. El Modelo de Propuesta de Conceptualización de Requisitos para Proyectos Software basados en formalismos de ingeniería de conocimiento que se presenta en la sección IV correspondiente a la Solución del problema identificado en esta sección, pretende realizar un aporte en este sentido. La solución surge como una versión facilitadora de realizar, de forma gradual, los pasos de interpretación y modelado del dominio, los cuales hasta la actualidad fueron dependientes de la experiencia del Ingeniero de Requisitos.

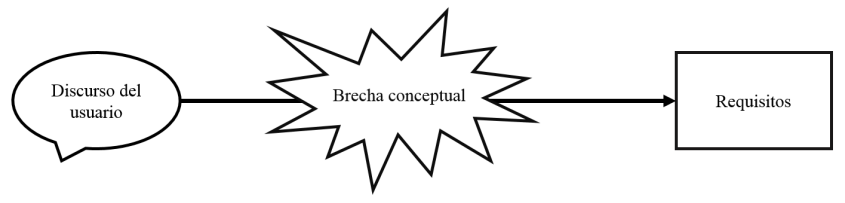

Fig. 5. Brecha conceptual que dificulta el proceso de conceptualización de requisitos para Proyectos software basados en formalismos de ingeniería de conocimiento.

\section{Sumario de investigación}

Pregunta 1: ¿Existen formalismos que permitan diferenciar subdominios de análisis que minimicen la brecha conceptual entre la educción de requisitos y el modelado conceptual? En caso afirmativo: ¿Cuáles?

Pregunta 2: En el caso de que existan tales formalismos, ¿Cuáles son las fases de dicho proceso, las tareas vinculadas a cada fase y las técnicas asociadas a cada tarea?

Se proponen soluciones a los interrogantes planteados y su correspondiente validación en la siguiente sección.

\section{SOLUCIÓN}

En esta sección se presenta la Propuesta de Conceptualización de Requisitos para Proyectos Software basados en formalismos de ingeniería de conocimiento (sección IV.A), las tareas asociadas a las técnicas del modelo de proceso (sección IV.B), los modelados no alcanzados por la propuesta (sección IV.C) y un resumen de la propuesta (sección IV.D).

\section{A. Modelo de Propuesta de Conceptualización de Requisitos para Proyectos Software}

En esta sección se presenta una propuesta de conceptualización de requisitos estructurada en dos partes: generalidades (sección IV.A.1), la Propuesta del Modelo (sección IV.A.2) y la estructura general del proceso de Conceptualización de Requisitos para Proyectos Software basados en formalismos de Ingeniería de Conocimiento (sección IV.A.3).

\section{1) Generalidades}

A partir del análisis efectuado en el capítulo anterior, se considera de interés citar nuevamente el problema abierto que aborda este artículo, recordando que el mismo se focaliza en la "brecha conceptual" presente en el proceso de educción de requisitos de usuario al proceso de modelado conceptual orientado a Proyectos Software [2]. Esta brecha conceptual dificulta la comprensión del problema manifestado por el usuario $y$, en consecuencia, constituye un escollo importante para la comprensión del problema manifestado por el usuario por parte del Ingeniero de Requisitos (de ahora en más IR) [8] [5] [9]. La figura 6 ilustra este concepto:

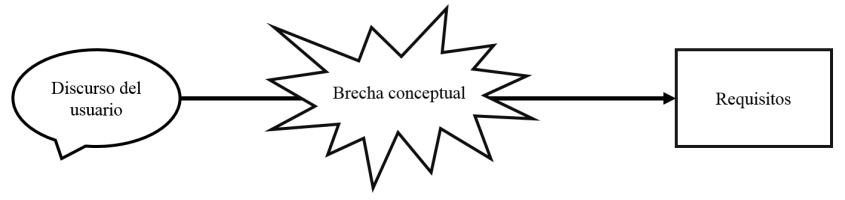

Fig. 6. Brecha conceptual que dificulta el proceso de conceptualización de requisitos para Proyectos software basados en formalismos de ingeniería de conocimiento.

Este artículo propone como solución, la inserción de una actividad de "Conceptualización de Requisitos para Proyectos Software basados en formalismos de Ingeniería de Conocimiento" que tiene como objetivo ser el nexo o conexión entre las actividades de educción de requisitos y modelado conceptual, facilitando la manera de comprensión del problema manifestado por el usuario. El alcance de dicha actividad está delimitado para proyectos software convencionales. En la figura 7 se puede observar la ausencia de la brecha conceptual, la cual se sustituye por la actividad de "Conceptualización de Requisitos para Proyectos Software". 


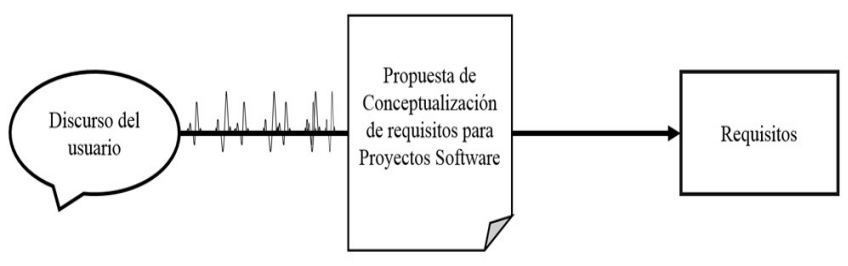

Fig.7. Propuesta de Conceptualización de Requisitos como nexo entre el Discurso del usuario y los Requisitos a educir.

La manera de pasar del discurso del usuario a los diagramas no se realiza bajo un procedimiento formalmente documentado y automatizado. Como consecuencia resulta ser una tarea engorrosa de realizar cuando el analista no posee suficiente experiencia o existen dificultades para reconocer clases, objetos, métodos, funciones y demás. Los formalismos utilizados para la solución son los descriptos en el apartado "Estado de la cuestión". Los formalismos de UML y de modelado de conocimiento no han sido modificados para la Propuesta de Conceptualización de Requisitos para Proyectos Software basados en formalismos de Ingeniería de Conocimiento, a excepción de las Tablas PER, que han sido adaptadas en tablas PERV (Palabras del experto con Vínculo). Se decidió expandir y adaptar a las tablas PERV, agregando el campo "Vínculo", ya que el modelado estándar no representa la relación entre la regla del experto y el actor que lleva a cabo la acción. A partir de esta modificación, se puede automatizar el armado del Diagrama de Casos de Uso.

Este artículo propone un proceso estándar con el fin de reducir esfuerzos, reducir tiempos, y estandarizar procesos donde la diversidad sea reducida. En las siguientes citas podemos comprobar la importancia de estandarizar en la ingeniería de software: "La estandarización de procesos provocará que de manera coordinada los procesos y esfuerzos se diseñen de forma común, es decir, todos los departamentos de la empresa o bien si se ella cuenta con otras localidades o centros de trabajo entenderán y verán los mismo, este lenguaje único permitirá mejorar la comunicación y dará soporte en todo momento a la toma de decisiones." [10].

2) Propuesta del Modelo de Conceptualización de Requisitos para Proyectos Software basados en formalismos de Ingeniería de Conocimiento.

El modelo de procesos está estructurado en dos fases con determinadas características que los componen:

- Análisis Orientado al Problema, cuyo objetivo es la comprensión del problema planteado por el usuario que concluye en la conceptualización del modelo de conocimiento para Proyectos Software. [2].

- Análisis Orientado al Producto, cuyo objetivo consiste en la obtención de los modelados que determinarán la manera de construir el sistema software en cuestión, dependiendo de lo analizado en la primera fase. [2].

La Propuesta de Conceptualización de Requisitos para Proyectos Software basados en formalismos de ingeniería de conocimiento está compuesta por dos Fases, donde la primera está integrada por cinco Tareas, la segunda por tres, y un conjunto de productos que pueden actuar como elementos de entrada y/o de salida de una determinada tarea. Todas las tareas requieren de ciertos productos para poder completarse, los cuales se procesan para proporcionar los correspondientes productos de salida. La fase de Análisis Orientado al Problema conlleva la primera tarea de la propuesta y es identificada bajo el nombre de Segmentación del Discurso de Usuario (S-DU), la cual necesita del Discurso de Usuario (DU) como producto de entrada y proporciona como producto de salida los correspondientes Segmentos de Texto (ST). Estos ST constituyen a su vez el producto de entrada para la realización de la tarea de Análisis Cognitivo de los Segmentos de Texto (ACST), la cual arroja como producto de salida la Tabla Concepto Atributo Valor (CAV). La tarea de Definición de las Palabras del Experto (D-PERV) poseen como producto de entrada los Segmentos de Texto (ST), y de la cual se obtiene como producto de salida las correspondientes Tablas Palabras Experto Regla Vínculo (PERV). La tarea de Confección de Diccionario (C-D) genera como producto de salida los Términos y las Definiciones del Diccionario (D) a partir de los Segmentos de Texto (ST) y los componentes de la Tabla Concepto Atributo Valor (CAV). La Técnica de Confección del Diagrama Jerárquico de Tareas (TC-DJT) requiere como producto de entrada las Reglas, Atributos y Valores (RAV) para la generación de los DJT. Luego comienza con el desarrollo de la fase de Análisis Orientado al Producto donde la primera tarea que se realiza es la Técnica de Armado del Diagrama de Casos de Uso (TA-DCU), que requiere como producto de entrada los Conceptos, Valores, Tareas y Vínculos (CVTV) representados mediante las Tablas Concepto Atributo Valor (CAV) y los Diagramas Jerárquicos de Tareas (DJT); la Técnica de Creación de Escenarios de Caso de Uso (TCECU) constituida por Nombre, Descripción, Señal, y Actores (NDSA); y la Técnica de Creación del Diagrama de Clases (TC-DC) que como producto de salida ofrecen las Clases, Objetos, Métodos y Relaciones (COMR) a partir de los Conceptos, Atributos, Valores, Tareas y Subtareas (CAVTSt) representados en las tablas CAV y los DJT.

\section{3) Estructura General del Proceso de Conceptualización de Requisitos para Proyectos Software}

Es esta sección se explica las fases, tareas y productos que componen la Estructura General del Proceso de Conceptualización de Requisitos para Proyectos Software basados en formalismos de Ingeniería de Conocimiento. En la tabla IV se ilustra la relación de dichos componentes. La Estructura pretende poner énfasis en la localización de cada uno de ellos dentro de la estructura del proceso y de cómo intervienen para que el mismo pueda llevarse a cabo. La Estructura General del Proceso de Conceptualización de Requisitos para Proyectos Software basados en formalismos de ingeniería de conocimiento se desarrolla por medio de la implementación de dos fases fundamentales: la fase de Análisis Orientado al Problema y la fase de Análisis Orientado al Producto. Para la realización de cada una de estas fases es necesario llevar a cabo una serie de tareas, las cuales tienen como función el procesamiento de ciertos productos de entrada para obtener los correspondientes productos de salida. En función de lo expuesto, para la fase de Análisis Orientado al Problema se tienen las siguientes relaciones entre las tareas y las técnicas que se deben aplicar: para el desarrollo de la tarea Segmentación del Discurso de Usuario (S-DU) se aplica la Técnica de Segmentación del Discurso de Usuario (TS - DU), para la implementación de la tarea Análisis Cognitivo de los Segmentos de Texto (ACST) se aplica la Técnica Cognitivas de Identificación de Conceptos Atributos y Valores (TC-CAV), para realizar la tarea de Definición de las Palabras del Experto (D- PERV) se aplica la Técnica Cognitiva de Identificación de Acciones Condiciones y Vínulos (TC-ACV), para la implementación 
TABLA IV. Fases TAREAS y Productos Del Proceso Propuesto

\begin{tabular}{|c|c|c|c|c|c|c|}
\hline \multirow{2}{*}{ FASE } & \multirow{2}{*}{ TAREA } & \multicolumn{2}{|c|}{ PRODUCTOS DE ENTRADA } & \multirow{2}{*}{$\begin{array}{c}\text { TECNICA DE } \\
\text { TRANSFORMACIÓx } \\
\text { A UTILIZAR }\end{array}$} & \multicolumn{2}{|c|}{ PRODUCTOS DE SALIDA } \\
\hline & & ENTRADA & REPRESENTACION & & SALIDA & REPRESENTACTON \\
\hline \multirow{5}{*}{$\begin{array}{l}\text { Análiais } \\
\text { Orientado } \\
\text { al } \\
\text { Problema }\end{array}$} & $\begin{array}{l}\text { Segmentación } \\
\text { del Discurso de } \\
\text { Usuario } \\
\text { (S-DU) }\end{array}$ & $\begin{array}{l}\text {-Discurso de } \\
\text { Usuario (DU) }\end{array}$ & -Texto Plano (TP) & $\begin{array}{l}\text { Técnica de } \\
\text { Segmentación del } \\
\text { Discurso de Usuario } \\
\text { (TS - DU) }\end{array}$ & $\begin{array}{l}\text {-Segmento de texto } \\
\text { (ST) }\end{array}$ & $\begin{array}{l}\text { Tabla de Segmentos de } \\
\text { Terto (T-ST) }\end{array}$ \\
\hline & $\begin{array}{l}\text { Análisis } \\
\text { Cognitivo de los } \\
\text { Segmentos de } \\
\text { Texto (ACST) }\end{array}$ & $\begin{array}{l}\text {-Segmentos de } \\
\text { Testo (ST) }\end{array}$ & $\begin{array}{l}\text {-Tablas de Segmento de } \\
\text { Texto (T-ST) }\end{array}$ & $\begin{array}{l}\text { Técnica Cognitiva de } \\
\text { Identificacion de } \\
\text { Conceptos Atributos y } \\
\text { Valores (TC-C.AV) }\end{array}$ & $\begin{array}{l}\text {-Conceptos } \\
\text {-Atributos } \\
\text {-Valores }\end{array}$ & $\begin{array}{l}\text { Tabla Concepto } \\
\text { Atributo Valor (CAV). }\end{array}$ \\
\hline & $\begin{array}{l}\text { Definición de } \\
\text { las Palabras } \\
\text { del Experto } \\
\text { (D-PERV) }\end{array}$ & $\begin{array}{l}\text {-Segmentos de } \\
\text { Testo (ST) } \\
\text {-Conceptos } \\
\text {-Atributos } \\
\text {-Valores }\end{array}$ & $\begin{array}{l}\text {-Tablas de Segmento de } \\
\text { Texto (T-ST) } \\
\text {-Tabla Concepto } \\
\text { Atributo Valor (CAV) }\end{array}$ & $\begin{array}{l}\text { Técnica Cognitiva de } \\
\text { Identificación de } \\
\text { Acciones Condiciones y } \\
\text { Vinculos (TC-ACV) }\end{array}$ & $\begin{array}{l}\text {-Acciones } \\
\text {-Condiciones } \\
\text {-Vinculos } \\
\text {-(ACV) }\end{array}$ & $\begin{array}{l}\text { Tablas Palabras } \\
\text { Experto Regla Vinculo } \\
\text { (PERV) }\end{array}$ \\
\hline & $\begin{array}{l}\text { Confección del } \\
\text { Diccionario } \\
\text { (C-D) }\end{array}$ & $\begin{array}{l}\text {-Segmentos de } \\
\text { Tento (ST) } \\
\text {-Conceptos } \\
\text {-Atributos } \\
\text {-Valores }\end{array}$ & $\begin{array}{l}\text {-Tablas de Segmento de } \\
\text { Texto (T-ST) } \\
\text {-Tabla Concepto } \\
\text { Atributo Valor (CAV) }\end{array}$ & $\begin{array}{l}\text { Técnica de Confección } \\
\text { del Diccionario (TC-D) }\end{array}$ & $\begin{array}{l}\text {-Definiciones } \\
\text {-Términos }\end{array}$ & Diccionario (D) \\
\hline & $\begin{array}{l}\text { Definición del } \\
\text { Diagrama } \\
\text { Jerarquico de } \\
\text { Tareas } \\
\text { (D-DJT) }\end{array}$ & $\begin{array}{l}\text {-Reglas } \\
\text {-Atributos } \\
\text {-Valores } \\
\text { (RAV) }\end{array}$ & $\begin{array}{l}\text {-Tablas Palabras } \\
\text { Experto Regla Vinculo } \\
\text { (PERV) } \\
\text {-Tabla Concepto } \\
\text { Atributo Valor (CAV) }\end{array}$ & $\begin{array}{l}\text { Técnica de Confección } \\
\text { del Diagrama } \\
\text { Jerárquico de Tareas } \\
\text { (TC-DJT) }\end{array}$ & $\begin{array}{l}\text { Dependencias y } \\
\text { Relaciones entre el } \\
\text { dominio del } \\
\text { problema. }\end{array}$ & $\begin{array}{l}\text { Dizgrama Jerárquico } \\
\text { de Tareas (DJT) }\end{array}$ \\
\hline \multirow{3}{*}{$\begin{array}{l}\text { Análisis } \\
\text { Orientado } \\
\text { al } \\
\text { Prociucto }\end{array}$} & $\begin{array}{l}\text { Definición de } \\
\text { los Diagramas } \\
\text { de Caso de Uso } \\
\text { (D-DCU) }\end{array}$ & $\begin{array}{l}\text {-Conceptos } \\
\text {-Valores } \\
\text {-Tareas } \\
\text {-Vinculos } \\
\text { (CVTV) }\end{array}$ & $\begin{array}{l}\text {-Tabla Concepto } \\
\text { Atributo Valor (CAV) } \\
\text {-Diagrama Jerárquico de } \\
\text { Tareas (DIT) }\end{array}$ & $\begin{array}{l}\text { Técnica de armado del } \\
\text { Diagrama de Casos de } \\
\text { Uso } \\
\text { (TA-DCU) }\end{array}$ & $\begin{array}{l}\text {-Actores } \\
-C \text { zsos } \\
(A C)\end{array}$ & $\begin{array}{l}\text { Diagrama de Casos de } \\
\text { Uso (DCU) }\end{array}$ \\
\hline & $\begin{array}{l}\text { Definición de } \\
\text { los Escenarios } \\
\text { de Caso de Uso } \\
\text { (D-ECU) }\end{array}$ & $\begin{array}{l}\text {-Tareas } \\
\text {-Actores } \\
\text {-Palabras del } \\
\text { experto }\end{array}$ & $\begin{array}{l}\text {-Tabla Concepto } \\
\text { Atributo Valor (CAV) } \\
\text {-Diagrama Jerárquico de } \\
\text { Tareas (DIT) } \\
\text { - Tablas Palabras } \\
\text { Experto Regla Vinculo } \\
\text { (PERV) } \\
\text {-Diagrama de Casos de } \\
\text { Uso (DCU). }\end{array}$ & $\begin{array}{l}\text { Técnica de Creación de } \\
\text { Escenarios de Casos de } \\
\text { Uso (TC-ECU) }\end{array}$ & $\begin{array}{l}\text {-Nombre } \\
\text {-Descripción } \\
\text {-Tipo de señal } \\
\text {-Actor } \\
\text { - Pasos } \\
\text {-Prioridad } \\
\text {-Riesgo }\end{array}$ & $\begin{array}{l}\text { Escenarios de Caso de } \\
\text { Uso (ECU). }\end{array}$ \\
\hline & $\begin{array}{l}\text { Definición del } \\
\text { Diagrama de } \\
\text { Clases (D-DC) }\end{array}$ & $\begin{array}{l}\text {-Conceptos } \\
\text {-Atributos } \\
\text {-Valores } \\
\text {-Tareas } \\
\text {-Subtareas }\end{array}$ & $\begin{array}{l}\text {-Tabla Concepto } \\
\text { Atributo Valor (CAV) } \\
\text {-Diagrama Jerárquico de } \\
\text { Tareas (DIT) }\end{array}$ & $\begin{array}{l}\text { Técrica de Creación del } \\
\text { Diagrama de Clases } \\
\text { (TC-DC) }\end{array}$ & $\begin{array}{l}\text {-Clases } \\
\text {-Objetos } \\
\text {-Métodos } \\
\text {-Relaciones }\end{array}$ & $\begin{array}{l}\text { Diagrama de Clases } \\
\text { (DC) }\end{array}$ \\
\hline
\end{tabular}

de la tarea de Confección del Diccionario (C-D) y para la tarea de Definición del Diagrama Jerárquico de Tareas, deberá implementarse la Técnica de Confección del Diagrama Jerárquico de Tareas (TC-DJT). En lo que respecta a la fase de Análisis Orientado al Producto se tienen las siguientes relaciones entre las tareas y las técnicas que se deben aplicar: para desarrollar la tarea de Definición de Diagrama de Casos de Uso (D-DCU) se aplica la Técnica de Armado del Diagrama de Casos de Uso (TA-DCU), para la implementación de la tarea de Definición de Escenarios de Caso de Uso (D- ECU) la técnica asociada es la Técnica de Creación de Escenarios de Casos de Uso (TC-ECU); y para la Definición del Diagrama de Clases (D-DC) la técnica asociada es la Técnica de Creación del Diagrama de Clases (TC-DC).

\section{B. Tareas asociadas a las Técnicas del Modelo de Proceso}

En esta sección se describe las tareas que le permiten al Ingeniero de Requisitos (IR) implementar las correspondientes técnicas que conforman el Proceso de Conceptualización de Requisitos para Proyectos Software basados en formalismos de IC segmentadas por las dos fases: Análisis Orientado al Problema y Análisis Orientado al Producto. A los efectos de ilustrar los entregables producidos en la propuesta, se presenta el caso de una tienda de alquiler de películas, con el fin de facilitar la comprensión de las etapas y la aplicación de las distintas técnicas utilizadas:

“...Descripción del Negocio y Problema de Negocio: Una tienda de alquiler de películas desea informatizar el registro de los DVD que posee. Cada uno de los vídeos tiene un número de cinta. Para cada película, el empleado deberá registrar el título, duración, director y la categoría según la siguiente clasificación: acción, terror, drama, suspenso, ficción, comedia y documental. Existen muchas copias de la mayoría de las películas, por lo tanto se les asignó a cada película un identificador específico. La tienda de video tiene muchos clientes y solamente alquila vídeos a personas que sean socias del vídeo club. Para que una persona pueda pertenecer al video club como socio, el interesado debe afiliarse en la página web, para lo cual se le asigna un número que lo identifica, nombres y apellidos, número telefónico, dirección de residencia. Para un alquiler, el empleado debe registrar la fecha de alquiler, día de devolución, cliente, y número de cinta...". 


\section{1) Tareas utilizadas en la Fase de Análisis Orientado al Problema.}

En esta sección se describen las tareas utilizadas para el desarrollo de las técnicas correspondientes a la fase de Análisis Orientado al Problema: la tarea de Segmentación del Discurso de Usuario (S - DU) (sección IV.B.1.a), la tarea de Análisis Cognitivo de los Segmentos de Texto (ACST) (sección IV.B.1.b), la tarea de Definición de las Palabras del Experto (D-PERV) (sección IV.B.1.c), la Confección del Diccionario (C-D) (sección IV.B.1.d) y la Definición del Diagrama Jerárquico de Tareas (TC-DJT) (sección IV.B.1.e).

\section{a) Tarea de Segmentación del Discurso de Usuario (S-DU)}

Esta tarea es la primera a implementar por el Ingeniero de Requisitos dentro de la fase de Análisis Orientado al Problema. Esta tarea está asociada a la Técnica de Segmentación del Discurso de Usuario (S-DU) [2]. Para aplicar la técnica (TS - DU), el IR cuenta con el Discurso del Usuario (DU) en lenguaje natural como producto de entrada, y comienza por una segmentación de dicho DU "frase" por "frase" (llamadas "frases cortas") y finalmente integrar estas frases y obtener los Segmentos de Texto (ST) que identifiquen situaciones de la realidad descripta del usuario. La técnica mencionada es representada en la Tabla V

TABLA V. TÉCNICA DE SEGMENTACIÓN DEL DISCURSO DEL USUARIO CON SUS ENTRADAS, SALIDAS Y PASOS

\begin{tabular}{|l|}
\hline \multicolumn{1}{|c|}{ Técnica de Segmentación del Discurso de Usuario (TS - DU) } \\
\hline Entrada: Discurso del Usuario (DU) \\
Salidas: Segmentos de Texto (ST) \\
\hline Paso 1: Segmentación del DU en "frases cortas" \\
Paso 2: Integración de las "frases cortas" en ST. \\
\hline
\end{tabular}

En este primer paso de esta tecnica se realiza un análisis preliminar del DU con el fin de generar "frases cortas". El concepto de segmentar el DU de esta forma proviene de la Ingeniería de Conocimiento y se basa en la técnica de Análisis de Protocolo para educir conocimiento de un experto cuando el mismo relata cómo resuelve un determinado problema [11] [12]; en este caso, en la fase de "Transcripción de protocolo", el ingeniero de conocimiento segmenta y transcribe el relato del experto en base a cuestiones tales como las pausas que realiza el experto en su exposición o las distintas entonaciones a lo largo de su relato. Esta segmentación inicial permite un tratamiento más simple del DU para afrontar el siguiente paso.

Las "frases cortas" constituyen el Segmento de Texto como producto de salida correspondiente a este paso.

En este paso se integran las "frases cortas" obtenidas en el paso anterior en ST descriptivos de una situación o episodio de la realidad. Estos ST están conformados por conjuntos de frases cortas y constituyen el subproducto de salida correspondiente a este paso. [2]. La tarea propuesta y los subproductos que se obtienen se pueden visualizar en la figura 8.

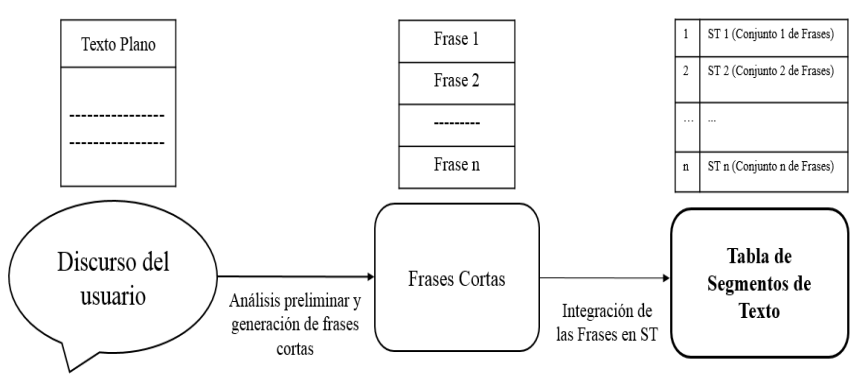

Fig. 8. Esquema y subproductos resultantes de aplicar la Técnica de Segmentación del Discurso de Usuario
Como resultado de aplicar la técnica definida al caso de estudio propuesto, se obtiene la tabla VI y la tabla VII. En la tabla VII se observan las frases cortas segmentadas a partir del discurso del usuario a partir de las pausas generadas y entonaciones del relato. En la tabla VIII se observa las frases cortas integradas en segmentos de texto.

TABLA VI. "FRASES CORTAS" RECONOCIDAS A PARTIR DEL DU.

\begin{tabular}{|c|l|}
\hline ID & Frases cortas \\
\hline FC1 & Una tienda de alquiler de películas desea informatizar el registro de los DVD que posee. \\
\hline FC2 & Cada uno de los vídeos tiene un número de cinta. \\
\hline FC3 & $\begin{array}{l}\text { Para cada película, el empleado deberá registrar el título, duración, director y la categoría } \\
\text { según la siguiente clasificación: acción, terror, drama, suspenso, ficción, comedia y } \\
\text { documental. }\end{array}$ \\
\hline FC4 & $\begin{array}{l}\text { Existen muchas copias de la mayoría de las películas, por lo tanto se les asignó a cada } \\
\text { película un identificador especifico. }\end{array}$ \\
\hline FC5 & $\begin{array}{l}\text { La tienda de video tiene muchos clientes y solamente alquila vídeos a personas que sean } \\
\text { socias del video club. }\end{array}$ \\
\hline FC6 & $\begin{array}{l}\text { Para que una persona pueda pertenecer al video club como socio, el interesado debe } \\
\text { afiliarse en la página web, para lo cual se le asigna un número que lo identifica, nombres y } \\
\text { apellidos, número telefónico, dirección de residencia. }\end{array}$ \\
\hline FC7 & $\begin{array}{l}\text { Para un alquiler, el empleado debe registrar la fecha de alquiler, día de devolución, cliente, } \\
\text { ynumero de cinta. }\end{array}$ \\
\hline
\end{tabular}

TABLA VII. TABLA DE ST A PARTIR DE LAS “FRASES CORTAS"

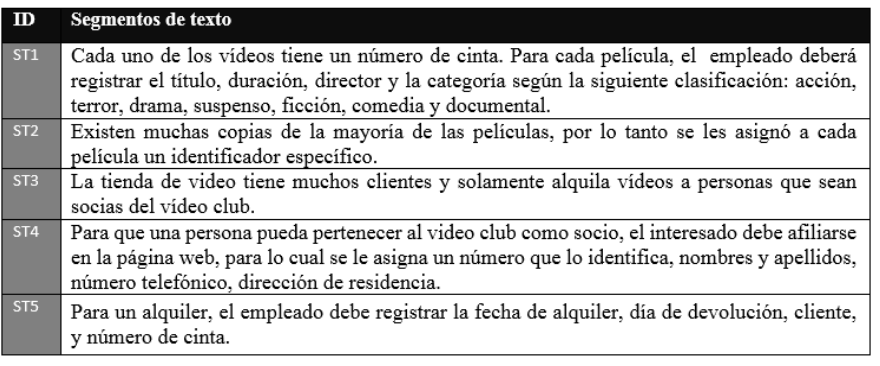

b) Tarea de Análisis Cognitivo de los Segmentos de Texto (ACST)

Por medio de esta tarea se implementa la segunda técnica que debe llevar a cabo el IR en la frase de Análisis Orientado al Problema, denominada Técnica Cognitiva de Identificación de Conceptos Atributos y Valores (TC-CAV). Para la aplicación de la TC-CAV el IR dispone como producto de entrada de cada uno de los ST que fueron obtenidos a partir de la aplicación de la técnica anterior (TS - DU); estos segmentos se procesan con la idea de identificar en los mismos, diferentes tipos de Conceptos, Atributos y Valores que serán representados en la Tabla Concepto Atributo Valor (CAV). Para comenzar a aplicar la (TC-CAV) el IR comienza por la identificación de Conceptos, Atributos y Valores en los ST para luego generar la Tabla CAV como producto de salida que proporciona la TC-CAV. La tabla VIII resume los pasos y procedimientos necesarios para la implementación de esta técnica.

TABLA VIII. TÉCNICA COGNITIVA DE IDENTIFICACIÓN DE ACTORES, ADJETIVOS Y VARIABLES CON SUS ENTRADAS, SALIDAS Y PASOS

Técnica Cognitiva de Identificación de Conceptos, Atributos y Valores (TC - CAV) Entrada: Tablas de Segmento de Texto (T-ST)

Salidas: Tabla Concepto Atributo Valor (CAV)

Paso 1: Análisis Cognitivo de los ST

1.1. Identificación de Conceptos en los ST

1.2. Identificación de Atributos en los ST

1.3. Identificación de Valores en los ST.

Paso 2: Generar la Tabla Concepto Atributo Valor (CAV)

A continuación se listan los pasos de la técnica:

En este primer paso se realiza el Análisis Cognitivo de los ST a los efectos de identificar los Conceptos, Atributos y Valores en cada uno de los ST obtenidos a partir de la aplicación de la técnica anterior. La realización de este paso se lleva a cabo por medio de los cuatro procedimientos, que se describen en los parrafos siguientes. 
Identificación de los Conceptos en los ST: por medio de este procedimiento se realiza el Análisis Cognitivo de los ST a los efectos de identificar los conceptos implicados en el texto. Los conceptos son los que protagonizarán el Software ya que interactúan con el sistema. Para cada ST debemos identificar personas físicas, con distintos tipos de roles, perfiles o cualquier palabra de significado similar. En este procedimiento deberán ser identificados los sustantivos que son determinantes en el núcleo del negocio, hacen al funcionamiento del sistema, con características particulares que lo describen. Constan de un estado, identidad y/o comportamiento. Son entidades provistas de un conjunto de atributos y reaccionan ante eventos. No necesariamente en todos los Segmentos del Texto exista actores o conceptos a relevar. Puede darse la circunstancia de que las personas físicas no sean conceptos propiamente dichos y el analista los ubique como Valor de lo que podría ser un Concepto "usuario" por ejemplo. Esta variación está sujeta a gusto del analista, siempre y cuando contemple una normalización adecuada de los elementos en cuestión. La identificación de variables o valores dentro de los ST son identificadas en el tercer procedimiento que veremos más adelante.

Identificación de los Atributos en los ST: por medio de este procedimiento se realiza el Análisis Cognitivo de los ST a los efectos de identificar los Atributos implicados en el texto. Los atributos son los que dan información relevante de los conceptos identificados en cada ST. Todos los conceptos poseen atributos, que a su vez contienen uno o más Valores. Esta es una manera de identificar un Concepto y de validar si están adecuadamente identificados. Este procedimiento consiste en identificar Atributos en los ST que complemente a los Conceptos para calificarlo, que exprese características o propiedades atribuidas al mismo.

Identificación de las Valores en los ST: por medio de este procedimiento se realiza el Análisis Cognitivo de los ST a los efectos de identificar los Valores implicados en el texto. El procedimiento consiste en reconocer para cada ST, los valores asociados a los atributos, tales como rangos numéricos, sitios, plazos, condicionales, adjetivos, u otros tipos de valores. Los distintos productos obtenidos constituyen el subproducto de salida correspondiente a este paso.

En este segundo paso se procede a generar la tabla CAV donde indique los diferentes tipos de conceptos, atributos y valores obtenidos en el total de los ST. Puede darse la circunstancia de que las ST no aporten datos suficientes debido a un Discurso de Usuario incompleto y nos demos cuenta al momento de modelar. Si se da la situación, deberíamos efectuar indagaciones posteriores para aclarar casos del sistema no contemplados. El subproducto que se obtiene se puede visualizar en la fig. 9.

Nota 1: En la mayoría de los casos, y siempre que el requerimiento solicite identificar cada Concepto, es aconsejable agregar como Atributo un "Id" que lo identifique unívocamente.

Nota 2: Cabe destacar que en la Tabla CAV no debemos ingresar acciones, las mismas serán desarrolladas en modelados posteriores.

Como resultado de aplicar la técnica definida al caso de estudio propuesto, se obtiene la tabla IX. En dicha tabla se observan los conceptos que interactúan con el sistema, personas físicas y sustantivos determinantes en el núcleo del negocio. También se observa los atributos que poseen cada uno de los conceptos describiéndolos, junto a los valores que pueden tomar, como por ejemplo, el tipo de categoría, ya sea: acción, terror, suspenso, drama, ficción, comedia o documental.

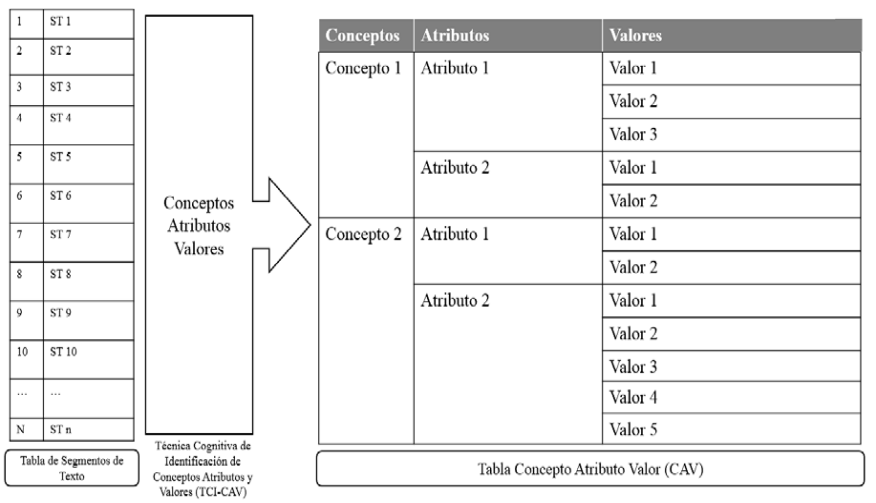

Fig. 9. Esquema y subproductos resultantes de aplicar la TC de Identificación de Conceptos, Atributos y Valores.

TABLA IX. TABLA CONCEPTO ATRIBUTO VALOR DEL CASO DE TIENDA DE VIDEO

\begin{tabular}{|l|l|l|}
\hline Concepto & Atributo & Valor \\
\hline \multirow{5}{*}{ Video } & IdCinta & Int \\
\cline { 2 - 3 } & Titulo & String \\
\cline { 2 - 3 } & Duración & Float \\
\cline { 2 - 3 } & Director & String \\
\cline { 2 - 3 } & & Acción \\
\cline { 2 - 3 } & \multirow{5}{*}{ Categoria } & Terror \\
\cline { 2 - 3 } & & Suspenso \\
\cline { 3 - 3 } & & Drama \\
\cline { 3 - 3 } & & Ficción \\
\cline { 2 - 3 } & & Comedia \\
\cline { 2 - 3 } & IdInteresado & Documental \\
\cline { 2 - 3 } & Nombre & Int \\
\cline { 2 - 3 } & Apellido & String \\
\cline { 2 - 3 } & Telefono & String \\
\cline { 2 - 3 } & Dirección & Int \\
\hline \multirow{5}{*}{ Empleado } & IdEmpleado & String \\
\cline { 2 - 3 } & Nombre & Int \\
\hline \multirow{5}{*}{} & FechaAlquiler & String \\
\cline { 2 - 3 } & FechaDevolución & Date \\
\cline { 2 - 3 } & IdCliente & Date \\
\cline { 2 - 3 } & IdCinta & Int \\
\cline { 2 - 3 } & IdAlquiler & Int \\
\hline \multirow{5}{*}{} & & Int \\
\hline
\end{tabular}

c) Tarea de Definición de las Palabras del Experto (DPERV)

Por medio de esta tarea se implementa la tercera técnica que debe llevar a cabo el IR en la frase "Análisis Orientado al Problema", denominada Técnica Cognitiva de Identificación de Acciones Condiciones y Vínculos (TCACV). Para la aplicación de la TC-ACV el IR dispone como productos de entrada de cada uno de los ST y la tabla CAV que fueron obtenidos a partir de la aplicación de la primera y segunda tarea del modelado; estos segmentos se procesan con la idea de identificar en los mismos, diferentes tipos de Acciones, Condiciones y Vínculos que serán representados en la Tabla Palabras del Experto Regla Vínculo (PERV). Este modelado es un derivado de la tabla PER [12]. Para comenzar a aplicar la Técnica Cognitiva de Identificación de Acciones Condiciones y Vínculos, el IR comienza por la identificación de acciones, condiciones y vínculos en los ST para luego generar las Tablas PERV como producto de salida. A continuación, en la siguiente tabla $(\mathrm{X})$ se puede observar un resumen de los pasos y procedimientos necesarios para la implementación de esta técnica, especificando productos de entrada y de salida:

TABLA X. TÉCNICA COGNITIVA DE IDENTIFICACIÓN DE 
ACCIONES CONDICIONES Y VÍNCULOS CON SUS ENTRADAS SALIDAS

Técnica Cognitiva de Identificación de Acciones Condiciones y Vínculos (TC - ACV) Entrada: Tablas de Segmento de Texto (T-ST) y Tablas Concepto Atributo Valor (CAV).

Paso 1: Análisis Cognitivo de los ST.

1.1. Identificación de las Acciones en los ST

12. Identificación de las Condiciones en los ST

1.2. Identificación de los Vínculos en los ST.

Paso 2: Generar la Tabla Palabras Experto Regla Vínculo (PERV)

En los párrafos siguientes se describen los pasos de la técnica.

En este primer paso se realiza el Análisis Cognitivo de los ST a los efectos de identificar las acciones, condiciones y vínculos en cada uno de los ST. La realización de este paso se lleva a cabo por medio de tres procedimientos, a saber.

Identificación de las acciones en los ST: por medio de este procedimiento se realiza el Análisis Cognitivo de los ST a los efectos de identificar las acciones implicados en el texto. El método consiste en extraer de los segmentos, todas las acciones posibles que permite el sistema, identificarlas por separado con un nombre dentro de lo definido como "Identificador de la Regla". Dichas acciones deberán ser plasmadas en el campo "Palabras del experto", del cual se deberá extraer textualmente del segmento.

Identificación de los condiciones en los ST: por medio de este procedimiento se realiza el Análisis Cognitivo de los ST a los efectos de identificar las condiciones implicadas en el texto, El método consiste en "traducir" a pseudocódigo lo definido en las "palabras del experto", tomando los elementos de la tabla CAV y de manera tal que usemos sentencias como: si, entonces, $<,>,=$, entre otros. Cuando la regla concluye en un valor que toma el sistema, los valores y/o atributos que serán parametrizados deberán estar formalmente documentado entre llaves " \{\} " y separados por punto y coma “;". Si la regla concluye en un elemento que el sistema devuelve, la manera de documentarlo es sin símbolos.

Identificación de vínculos en los ST: por medio de este procedimiento se realiza el Análisis Cognitivo de los ST a los efectos de identificar los vínculos implicados en el texto. El procedimiento consiste en identificar el perfil/es o rol/es que están asociados a las acciones reconocidas en el punto 1.1. El método consiste en analizar el verbo de cada acción y preguntarse a uno mismo, quién es el sujeto que realiza la acción. Los distintos productos obtenidos constituyen el subproducto de salida correspondiente a este paso.

El segundo paso es generar las tablas Palabras Experto Regla Vínculo (PERV) donde indique las diferentes acciones como "palabras del experto", identificadas unívocamente mediante un título dentro del campo "Identificador de la regla", y asociada a un pseudocódigo que describa la condición de cada acción. Cada regla deberá poseer uno o más vínculos dependiendo del caso. A modo de ejemplo genérico, el subproducto que se obtiene a partir de la aplicación de esta técnica se puede visualizar en la tabla XI.

TABLA XI. TABLA PALABRA EXPERTO REGLA VÍNCULO (PERV)

\begin{tabular}{|l|l|}
\hline Identificador de la regla & \multicolumn{1}{|c|}{ Nombre de la regla } \\
\hline Palabras del experto & ..."Acción "... \\
\hline Regla & $\begin{array}{l}\text { SI Condicion1 Y } \\
\text { Condicion2 } \\
\text { ENTONCES } \\
\text { Valor1 } \leftarrow \text { Valor1 }+ \text { Valor2 }\end{array}$ \\
\hline Vinculo & \multicolumn{1}{|c|}{ Sujeto asociado a la acción. } \\
\hline
\end{tabular}

Como resultado de aplicar la técnica definida al caso de estudio propuesto, se obtiene la tabla XII, XIII y XIV. En estas Salidas: Tabla Palabras Experto Regla Vínculo(PERV) y en relación a las acciones identificadas en el punto anterior.

tablas se observan las palabras del experto identificada en los $\mathrm{ST}$, con el identificador de la regla y los vínculos, ya sea empleado o interesado.

\section{TABLA XII. REGLA PERV “REGISTRAR VIDEO”}

\begin{tabular}{|l|l|}
\hline Identificador de la regla & \multicolumn{1}{c|}{ REGISTRAR VIDEO } \\
\hline Palabras del experto & $\begin{array}{c}\text {..."Cada uno de los vídeos tiene un número de cinta. Para cada pelicula, el } \\
\text { empleado deberá registrar el título, duración, director y la categoria según la } \\
\text { siguiente clasificación: acción, terror, drama, suspenso, ficción, comedia y } \\
\text { documental" ... }\end{array}$ \\
\hline Regla & $\begin{array}{l}\text { SI EMPLEADO } \leftarrow \text { IDEMPLEADO } \\
\text { ENTONCES } \\
\text { VIDEO } \leftarrow \text { \{idCinta; titulo; duración; director; categoría }\}\end{array}$ \\
\hline Vinculo & \multicolumn{1}{|c|}{ EMPLEADO } \\
\hline
\end{tabular}

TABLA XIII. REGLA PERV “AFILIACIÓN CLIENTE”

\begin{tabular}{|c|c|}
\hline Identificador de la regla & AFILIACIÓN CLIENTE \\
\hline Palabras del experto & $\begin{array}{c}\text {... "Para que una persona pueda pertenecer al video club como socio, el } \\
\text { interesado debe afiliarse en la página web, para lo cual se le asigna un número } \\
\text { que lo identifica, nombres y apellidos, número telefónico, dirección de } \\
\text { residencia"... }\end{array}$ \\
\hline Regla & $\begin{array}{l}\text { SI INTERESADO } \leftarrow \text { IDINTERESADO } \\
\text { ENTONCES } \\
\text { CLIENTE } \leftarrow \text { \{IdInteresado; nombre; apellido; teléfono; dirección }\}\end{array}$ \\
\hline Vinculo & INTERESADO \\
\hline
\end{tabular}

TABLA XIV. REGLA PERV “ALQUILER DE VIDEO”

\begin{tabular}{|c|c|}
\hline Identificador de la regla & ALQUILER DE VIDEO \\
\hline Palabras del experto & $\begin{array}{l}\text {... "Para un alquiler, el empleado debe registrar la fecha de alquiler, dia de } \\
\text { devolución, cliente, y número de cinta" ... }\end{array}$ \\
\hline Regla & $\begin{array}{l}\text { SI EMPLEADO } \leftarrow \text { IDEMPLEADO } \\
\text { ENTONCES } \\
\text { ALQUIIER } \leftarrow \text { idCinta; idAlquiler, IdCliente; FechaDevolucion; } \\
\text { FechaAlquiler }\}\end{array}$ \\
\hline Vínculo & EMPLEADO \\
\hline
\end{tabular}

d) Tarea de Confección del Diccionario (C-D)

Mediante esta tarea se implementa la cuarta técnica que debe llevar a cabo el IR en la frase de Análisis Orientado al Problema, denominada Técnica de Confección del Diccionario (TC-D). Para la aplicación de la TC-D, el IR dispone como producto de entrada cada uno de los ST, y los Conceptos Atributos y Valores (CAV) que conformaron la Tabla CAV. Estos productos se procesan con la idea de poder ser definidos y representados en un Diccionario. Para comenzar a aplicar la TC-D, el IR comienza por la creación de una tabla con los campos "Término" y "Definición". La Tabla XV resume los pasos y procedimientos necesarios para la implementación de esta técnica.

\section{TABLA XV. TÉCNICA DE CONFECCIÓN DEL DICCIONARIO CON SUS ENTRADAS, SALIDAS Y PASOS}

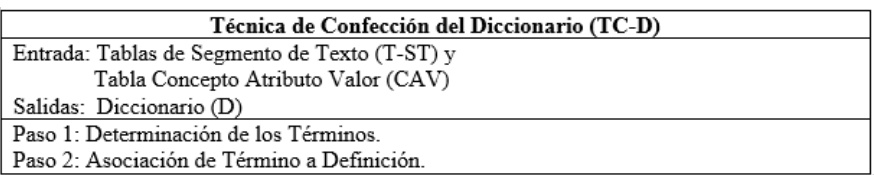

En los párrafos siguientes se describen los pasos de la técnica.

En este paso se deberá extraer todos los Conceptos, Atributos y Valores de la Tabla CAV generada y asignarlas como Términos, con el fin de poder identificar las definiciones de cada una.

En este segundo paso, debemos identificar cada uno de los Términos del Diccionario en las Tablas de Segmento de Texto y extraer de los ST la información que releve al mismo, ya sea describiéndolo. En los casos donde el ST no aporte información puntual del término, por ejemplo, en los casos donde sean valores "Si" o "No", la descripción estará sujeta al entendimiento del IR. Esas descripciones serán las Definiciones que se obtienen como producto de salida al 
aplicar esta técnica. El producto de salida se representará tal como lo indica la Tabla XVI.

TABLA XVI. DICCIONARIO

\begin{tabular}{|l|l|}
\hline Término & Definición \\
\hline Atributo & ST que describe al atributo de la fila. \\
\hline Concepto & ST que describe al concepto de la fila. \\
\hline Valor & ST que describe al valor de la fila. \\
\hline Valor 2. & ST que describe al valor 2 de la fila. \\
\hline Valor 3. & ST que describe al valor 3 de la fila. \\
\hline Valor 4. & ST que describe al valor 4 de la fila. \\
\hline Valor 5. & ST que describe al valor 5 de la fila. \\
\hline Valor $n$. & ST que describe al valor $n$ de la fila. \\
\hline
\end{tabular}

Como resultado de aplicar la técnica definida al caso de estudio propuesto, se obtiene la tabla XVII. En dicha tabla se observan los conceptos, atributos y valores en la columna "términos" y los segmentos de texto que describen a los mismos en la columna "definición".

\section{TABLA XVII. DICCIONARIO DE TIENDA DE VIDEO}

\begin{tabular}{|l|l|}
\hline Término & Definición \\
\hline Alquiler & Transacción que se efectúa como núcleo del negocio. \\
\hline Apellido & Apellido del cliente o interesado. \\
\hline Categoría & Videos según la clasificación: acción, terror, drama, suspenso, etc. \\
\hline Date & Valor que toman los atributos relacionados a fechas. \\
\hline Dirección & Dirección del cliente o interesado. \\
\hline Director & Director del video. \\
\hline Duración & Duración del video. \\
\hline Empleado & Personal de la tienda de video. \\
\hline FechaAlquiler & Fecha que se alquiló el video. \\
\hline FechaDevolución & Fecha que deberá ser devuelto el video. \\
\hline Float & Valor que representa un número. \\
\hline IdAlquiler & Identificación unívoca y numerada que hace referencia a un alquiler especifico. \\
\hline IdCinta & Identificación unívoca y numerada que hace referencia a una copia de video. \\
\hline IdCliente & Identificación univoca y numerada que hace referencia a un cliente o interesado. \\
\hline IdEmpleado & Identificación univoca y numerada que hace referencia al empleado. \\
\hline IdInteresado & Identificación unívoca y numerada que hace referencia a un interesado o cliente. \\
\hline Int & Valor que representa un entero. \\
\hline Interesado & Persona que desea convertirse en cliente de la tienda. \\
\hline Nombre & Nombre de la persona. \\
\hline String & Cadena de caracteres que puede representar como valor de un atributo. \\
\hline Telefono & Teléfono del cliente o interesado. \\
\hline Titulo & Nombre del video. \\
\hline Video & Producto que comercializa la tienda en cuestión \\
\hline
\end{tabular}

\section{e) Tarea de Definición del Diagrama Jerárquico de}

\section{Tareas (D-DJT)}

Por medio de esta tarea se implementa la última técnica que debe efectuar el IR en la fase de Análisis Orientado al Problema, denominada Técnica de Confección del Diagrama Jerárquico de Tareas (D-DJT). Para la aplicación de la (TCDJT) el IR dispone como producto de entrada de Reglas, Atributos y Valores (RAV) que son extraídos de las Tablas PERV y CAV creadas anteriormente. Para aplicar la (TC-DJT) el IR deberá considerar los siguientes pasos especificados a continuación. La Tabla XVIII resume los lineamientos, las entradas y salidas para la implementación de esta técnica.

TABLA XVIII. TÉCNICA DE ARMADO DEL DIAGRAMA DE CASOS DE USO CON SUS ENTRADAS Y SALIDAS.

\begin{tabular}{|l|}
\hline \multicolumn{1}{|c|}{ Técnica de Confección del Diagrama Jerárquico de Tareas (TC - DJT) } \\
\hline Entrada: Tabla Concepto Atributo Valor (CAV) \\
Tablas Palabras Experto Regla Vínculo (PERV) \\
Salidas: Diagrama Jerárquico de Tareas (DJT). \\
\hline $\begin{array}{l}\text { Pasos que constan en una numeración correlativa de tareas, vinculación a sub tareas, relación } \\
\text { con reglas PERV y constitución para la construcción del DJT. }\end{array}$ \\
\hline
\end{tabular}

En los párrafos siguientes se describen los pasos de la técnica.

Todas las tareas creadas deberán estar enumeradas en orden lógico de transacción.
En caso de ser necesario, las tareas pueden estar vinculadas a sub tareas. Estas últimas son diseñadas en el caso de que una tarea dependa de otra para llevarse a cabo.

En lo que respecta a la creación y reconocimiento de tareas, éstas se vincularán a la cantidad de reglas en las Tablas PERV, portando el mismo nombre para cada una de las reglas.

Cada una de las tareas deberá constar de "Precondiciones" y "Post condiciones": El primero consiste en ingresar como Parámetros de Entrada, los Atributos que requieren para alcanzar el logro de la tarea en cuestión. El segundo conforma los Atributos asociados a Valores que devuelve el sistema para que la Tarea pueda concluir.

Cuando el IR crea necesario modelar una tarea asociada a sub tareas, deberá crearse un árbol de la siguiente manera: Una tarea posee como nodo raíz la función principal que es a lo que deberá llegar el sistema. Las sub tareas son nodos hijos que requieren ser numeradas según la nomeclatura del nodo raíz. La precondición de la Sub tarea n es la post condición de la sub tarea n-1, sucesivamente en el caso de existir más situaciones. A su vez, la Post Condición es la sub tarea n es la precondición de la tarea raíz. No necesariamente se deba escribir las post condiciones en la precondición siguiente, sino que pueden estar implícitas.

También puede darse el caso de que existan atributos que se agreguen como precondiciones a las post condiciones de la tarea anterior. El recorrido del árbol creado deberá leerse conforme al siguiente algoritmo:

- Postorden: (izquierdo, derecho, raíz). Para recorrer un árbol no vacío en post orden, hay que realizar las siguientes operaciones recursivamente en cada nodo:

- Atraviese el sub-árbol izquierdo

- Atraviese el sub-árbol derecho

- Visite la raíz

La representación del producto de salida es el Diagrama Jerárquico de Taras (DJT), conformado por Dependencias y Relaciones entre el dominio del problema. El subproducto que se obtiene se puede visualizar en la figura 10.

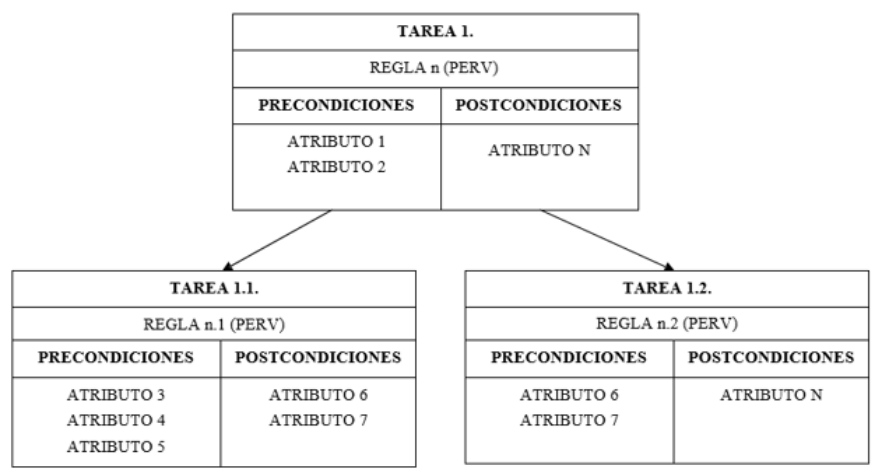

Fig. 10. Representación de un modelo de Diagrama Jerárquico de Tareas.

Como resultado de aplicar la técnica definida al caso de estudio propuesto, se obtiene la figura 11. En dicha figura se observa el Diagrama Jerárquico de Tareas, compuesto por las tareas enumeradas y definidas según las reglas PERV del modelado. Adjunto a cada tarea se observa los atributos identificados como precondiciones y post condiciones.
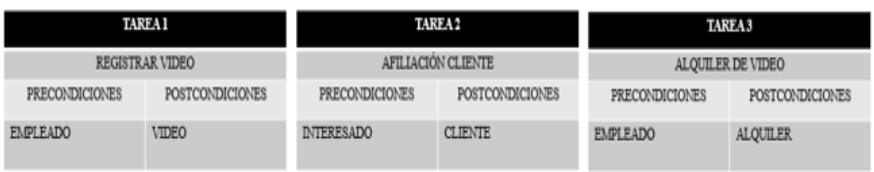
Fig. 11. Diagrama Jerárquico de Tareas Tienda de Video.

\section{2) Tareas utilizadas en la Fase de Análisis Orientado al Producto.}

En esta sección se describen las tareas utilizadas para el desarrollo de las técnicas correspondientes a la fase de Análisis Orientado al Producto: la Tarea de Definición del Diagrama de Casos de Uso (D-DCU) (sección IV.B.2.a), la Tarea de Definición de los Escenarios de Caso de Uso (DECU) (sección IV.B.2.b) y la Tarea de Definición del Diagrama de Clases (D-DC) (sección IV.B.2.c).

a) Tarea de Definición del Diagrama de Casos de Uso (D-DCU).

Esta tarea es la primera a implementar por el Ingeniero de Requisitos dentro de la fase de Análisis Orientado al Producto. La técnica asociada a esta tarea es denominada Técnica de Armado del Diagrama de Casos de Uso (TADCU) [13]. Para aplicar la técnica (TA - DCU), el IR cuenta con los Conceptos, Valores, Tareas y Vínculos (CVTV) como productos de entrada, extraídos de los modelados en la fase anterior. La Tabla XIX resume los pasos y procedimientos necesarios para la implementación de esta técnica.

TABLA XIX. TÉCNICA DE ARMADO DEL DIAGRAMA DE CASOS DE USO CON SUS ENTRADAS SALIDAS Y PASOS

\begin{tabular}{|l|}
\hline \multicolumn{1}{|c|}{ Técnica de Armado del Diagrama de Casos de Uso (TA - DCU) } \\
\hline Entrada: Tabla Concepto Atributo Valor (CAV) \\
Diagrama Jerárquico de Tareas (DJT). \\
Salidas: Diagrama de Casos de Uso (DCU). \\
\hline Paso 1: Identificación de Actores y Casos (AC) \\
Paso 2: Asociación de Actores a los Casos. \\
\hline
\end{tabular}

En los párrafos siguientes se describen los pasos de la técnica.

En este primer paso se identifican los actores del Diagrama. Primero deberá extraerse los Conceptos de la Tabla CAV creada anteriormente. Los Conceptos que se definirán como actores son aquellos que interactúen con el sistema a desarrollar, es decir, distinguir los entes activos de los pasivos. Entiéndase que actores activos desempeñan funciones operativas. Como hemos mencionado en la sección IV.B.1.2, existen situaciones donde personas físicas son asignadas como Valores dentro de un mismo Concepto con el fin de simplificar el modelado de la Tabla CAV. En esos casos deberá tomarse esos "Valores" como Actores del Diagrama. Por otro lado, las tareas del DJT son las que deberán extraerse para identificar los Casos del DCU.

En este segundo paso se especifica la asociación que deberá realizarse entre Actores y Casos. Para realizar esta vinculación, debemos inspeccionar en las tablas PERV generadas, qué vinculo (en este caso Actor), se corresponde con cada caso de uso y relacionarlo mediante una flecha. El subproducto que se obtiene se puede visualizar en la figura 12 .

Como resultado de aplicar la técnica definida al caso de estudio propuesto, se obtiene la figura 13. En dicha figura se observa el Diagrama de Casos de Uso, compuesto por actores y casos. Se definió a "empleado" e "interesado" como actores por ser conceptos que interactúan con el sistema a desarrollar, con características que los definen como actores activos.

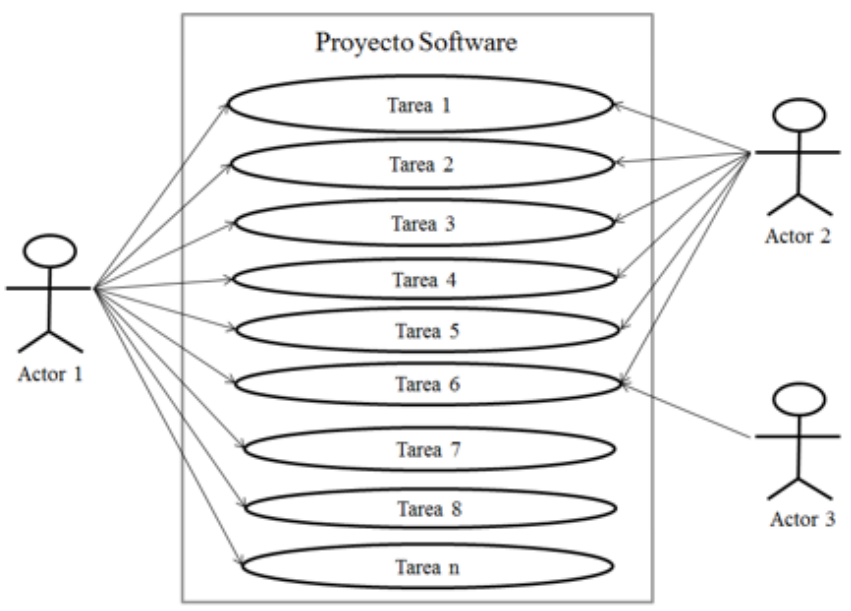

Fig.12. Diagrama de Casos de Uso (DCU)

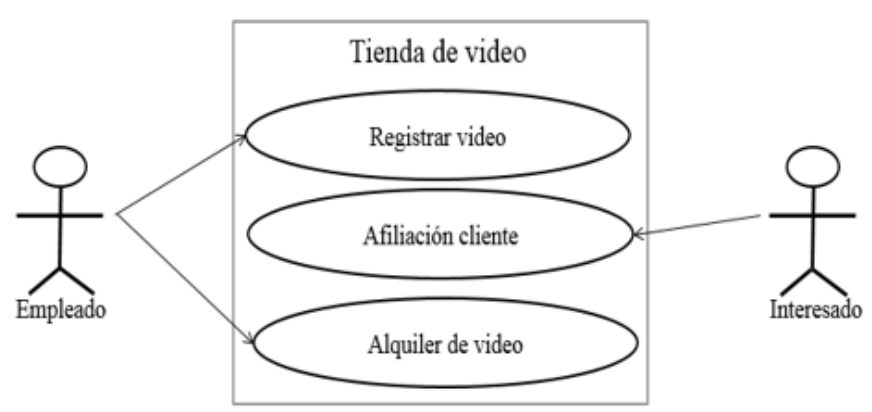

Fig. 13. Diagrama de Casos de Uso Tienda de Video

b) Tarea de Definición de los Escenarios de Caso de Uso (D-ECU).

Por medio de esta tarea se implementa la última técnica que debe llevar a cabo el IR en la frase de Análisis Orientado al Producto, denominada Técnica de Creación de Escenarios de Casos de Uso (TC-ECU) [13]. Para la aplicación de la TCECU el IR dispone como producto de entrada Tareas, Actores y Palabras del Experto que fueron obtenidos a partir de la aplicación de las técnicas de la fase Análisis Orientado al Problema. Estos productos se procesan con la idea de identificar el nombre del escenario, la Descripción, el Tipo de señal y Actor que serán representados en los Escenarios de Caso de Uso (ECU). Para aplicar la (TC-ECU) el IR deberá seguir los pasos descriptos a continuación. La Tabla XX resume los lineamientos, las entradas y salidas implicadas para la implementación de esta técnica.

TABLA XX. TÉCNICA DE CREACIÓN DE ESCENARIOS DE CASOS DE USO CON SUS ENTRADAS Y SALIDAS

\begin{tabular}{|l|}
\hline \multicolumn{1}{|c|}{ Técnica de Creación de Escenarios de Casos de Uso (TC-ECU) } \\
\hline Entrada: Tabla Concepto Atributo Valor (CAV) \\
Diagrama Jerárquico de Tareas (DJT) \\
Tablas Palabras Experto Regla Vínculo (PERV) \\
Diagrama de Casos de Uso \\
Salidas: Escenarios de Caso de Uso (ECU). \\
\hline $\begin{array}{l}\text { Pasos que constan en formalismos para la creación de escenarios de caso de uso y una guia } \\
\text { para establecer relaciones con los modelados efectuados en tareas anteriores. }\end{array}$ \\
\hline
\end{tabular}

En los párrafos siguientes se describen los pasos de la técnica.

Deberá existir un escenario por cada tarea y/o subtarea del DJT. El Nombre del Caso de Uso deberá llevar la misma identificación que la tarea o subtarea asociada, obteniendo una numeración única y correlativa para la distinción de escenarios generados. El campo Área llevará el nombre completo del Proyecto Software en cuestión.

Para identificar Actores en cada ECU debemos observar el Diagrama de Casos de Uso para reconocer el Actor que se 
vincula a la tarea o subtarea del mismo. El campo Descripción deberá poseer las Palabras del Experto que describe la tarea o subtarea del DJT. El objetivo de este parámetro es dar más detalle del alcance de este Caso de uso. El campo "Activar evento" describe el procedimiento y detalla el botón a ejecutar para activar la operación. La Señal de cada escenario podrá ser externa (donde el usuario es el predecesor de la transacción involucrada), o interna (donde el sistema es el predecesor del proceso). Definir el tipo de señal del escenario es posible al observar el Actor del Caso de Uso. Si el Actor es parte del software, la señal es interna. Caso contrario, la señal es externa. Los campos denominados "Pasos desempeñados" e "Información de los Pasos" no surgen a partir de la Propuesta de Conceptualización de Requisitos sino que son producto del IR a partir de todo lo relevado. Las precondiciones estarán definidas al comienzo de las reglas PERV, donde se detalla que regla debe darse para entrar en el bucle de la condición. Las "Postcondiciones" son el resultado de la operatoria en cuestión. El campo "Suposiciones" describe lo que deberá existir para que se llegue al objetivo. Por ejemplo: un navegador web, clave de usuario, contraseña válida, entre otros. El campo "Reunir requerimientos" describe lo que permite hacer esa acción. eberá preguntarse el IR, que se tendrá que controlar, limitar, $\mathrm{u}$ otro tipo de validación que considere como "Aspectos sobresalientes". La Prioridad y Riesgo son conceptos del ECU que oscilan en los siguientes valores:

Alta: Derivan de Escenarios de Casos de Uso compuestos por Tareas críticas en actividades del Proyecto Software, con una cantidad de pasos extensa y varios actores implicados.

Media: Derivan de Escenarios de Casos de Uso compuestos por Tareas de consulta en el Proyecto Software.

Baja: Derivan de Escenarios de Casos de Uso compuestos por Subtareas y/o Tareas que no realizan actividades críticas en Proyecto Software.

Un ejemplo del subproducto que se obtiene se puede visualizar en la tabla XXI.

TABLA XXI. ESCENARIO DE CASO DE USO A PARTIR DE IMPLEMENTAR LA TÉCNICA TC-ECU

\begin{tabular}{|c|c|}
\hline Nombre del Caso de Uso: & Tarea/Subtarea n del DJT \\
\hline Área: & Nombre del Proyjecto Software \\
\hline Actor: & Actor del Diagrama de Caso de Uso \\
\hline Descripción: & Palabra del experto vinculada a la tarea $n$. \\
\hline Activar evento: & $\begin{array}{l}\text { Explicación de la palabra del experto concluyendo en un } \\
\text { evento activador ya sea un botón. }\end{array}$ \\
\hline Tipo de señal: & Externa/Interna \\
\hline Pasos desempeñados & Información para los pasos \\
\hline 1) Paso 1 & Se describe el paso 1 . \\
\hline 2) Paso 2 & Se describe el paso 2. \\
\hline 3) Paso 3 & Se describe el paso 3. \\
\hline n) Paso $\mathrm{n}$ & Se describe el paso $\mathrm{n}$. \\
\hline Precondiciones: & $\begin{array}{l}\text { Se define la/s condición/es para desempeñar el caso de } \\
\text { uso en cuestión. }\end{array}$ \\
\hline Poscondiciones: & Objetivo conseguido al aplicar los pasos. \\
\hline Suposiciones: & Tiene un navegador, una clave, una contraseña, etc. \\
\hline Reunir requerimientos: & Beneficios de aplicar este caso de uso. \\
\hline Aspectos sobresalientes: & Ej: ¿Se debe controlar el número de intentos? \\
\hline Prioridad: & Alta - Media - Baja \\
\hline Riesgo: & Alta - Media - Baja \\
\hline
\end{tabular}

Como resultado de aplicar la técnica definida al caso de estudio propuesto, se obtienen las tablas XXII, XXIII y XXIV. En dichas tablas se observan los Diagramas de Escenario de Casos de Uso.
TABLA XXII. ESCENARIO DE CASO DE USO "REGISTRAR VIDEO"

\begin{tabular}{|c|c|c|}
\hline Nombre del Caso de Uso: & Registrar Video & ECU: 1 \\
\hline$\overline{\text { Área: }}$ & \multicolumn{2}{|c|}{ Sistema de Tienda de Video } \\
\hline Actor: & \multicolumn{2}{|c|}{ Empleado } \\
\hline Descripción: & \multicolumn{2}{|c|}{$\begin{array}{c}\text {... "Cada uno de los vídeos tiene un número de cinta. Para } \\
\text { cada película, el empleado deberá registrar el título, } \\
\text { duración, director y la categoría según la siguiente } \\
\text { clasificación: acción, terror, drama, suspenso, ficción, } \\
\text { comedia y documental"... }\end{array}$} \\
\hline Activar evento: & \multicolumn{2}{|c|}{$\begin{array}{l}\text { E1 empleado, una vez que ingresa al módulo de "Ingresar } \\
\text { nuevo título", carga los datos correspondientes y luego } \\
\text { presiona el botón "Ejecutar". }\end{array}$} \\
\hline Típo de señal: & \multicolumn{2}{|c|}{ Externa } \\
\hline Pasos desempeñados & \multicolumn{2}{|c|}{ Información para los pasos } \\
\hline 1) Registrar video & \multicolumn{2}{|c|}{$\begin{array}{l}\text { Ingresa al módulo de "Ingresar nuevo título", y define } \\
\text { título, duración, director, y categoría. }\end{array}$} \\
\hline 2) Ejecutar confirmación & \multicolumn{2}{|c|}{$\begin{array}{l}\text { Luego, el empleado presiona el botón "Ejecutar" para dar el } \\
\text { alta definitiva del video. }\end{array}$} \\
\hline Precondiciones: & \multicolumn{2}{|c|}{$\begin{array}{l}\text { El empleado realiza la identificación positiva para acceder a } \\
\text { las funcionalidades del software. }\end{array}$} \\
\hline Poscondiciones: & \multicolumn{2}{|c|}{ E1 empleado da el alta del video. } \\
\hline Suposiciones: & \multicolumn{2}{|c|}{$\begin{array}{l}\text { E1 empleado tiene un usuario, contraseña y el sistema instalado } \\
\text { en el ordenador. }\end{array}$} \\
\hline Reunir requerimientos: & \multicolumn{2}{|c|}{ Esta acción permite dar el alta de un video en el sistema. } \\
\hline Aspectos sobresalientes: & \multicolumn{2}{|c|}{ Generar un log de las transacciones efectuadas por el usuario. } \\
\hline Prioridad: & \multicolumn{2}{|c|}{ Alta } \\
\hline Riesgo: & \multicolumn{2}{|c|}{ Alta } \\
\hline
\end{tabular}

TABLA XXIII. ESCENARIO DE CASO DE USO "AFILIACIÓN CLIENTE"

\begin{tabular}{|c|c|c|}
\hline Nombre del Caso de Uso: & Afiliación cliente & ECU: 2 \\
\hline Área: & \multicolumn{2}{|c|}{ Sistema de Tienda de Video } \\
\hline Actor: & \multicolumn{2}{|c|}{ Interesado } \\
\hline Descripción: & \multicolumn{2}{|c|}{$\begin{array}{l}\text {... "Para que una persona pueda pertenecer al video club } \\
\text { como socio, el interesado debe afiliarse en la página web, para } \\
\text { lo cual se le asigna un número que lo identifica, nombres y } \\
\text { apellidos, número telefónico, dirección de residencia"... }\end{array}$} \\
\hline Activar evento: & \multicolumn{2}{|c|}{$\begin{array}{l}\text { El interesado, una vez que ingresa al módulo de "Afiliación", } \\
\text { carga los datos correspondientes y luego presiona el botón } \\
\text { "Ejecutar". }\end{array}$} \\
\hline Tipo de señal: & \multicolumn{2}{|c|}{ Externa } \\
\hline Pasos desempeñados & \multicolumn{2}{|c|}{ Información para los pasos } \\
\hline 1) Afiliación & \multicolumn{2}{|c|}{$\begin{array}{l}\text { Ingresa al módulo de "Afiliación", carga los datos } \\
\text { personales en los campos obligatorios. }\end{array}$} \\
\hline 2) Ejecutar confirmación & \multicolumn{2}{|c|}{$\begin{array}{l}\text { Luego, el interesado presiona el botón "Ejecutar" para } \\
\text { ejecutar la transacción de alta. }\end{array}$} \\
\hline Precondiciones: & \multicolumn{2}{|c|}{$\begin{array}{l}\text { E1 interesado no debe ser cliente ni empleado de la tienda } \\
\text { de videos. }\end{array}$} \\
\hline Poscondiciones: & \multicolumn{2}{|c|}{ El interesado se convierte en cliente socio. } \\
\hline Suposiciones: & \multicolumn{2}{|c|}{$\begin{array}{l}\text { E1 interesado tiene una conexión a internet y acceso al sitio } \\
\text { web por medio de un navegador. }\end{array}$} \\
\hline Reunir requerimientos: & \multicolumn{2}{|c|}{ Esta acción permite dar de alta clientes. } \\
\hline Aspectos sobresalientes: & \multicolumn{2}{|c|}{ Generar un log de las transacciones efectuadas por el usuario. } \\
\hline Prioridad: & \multicolumn{2}{|c|}{ Alta } \\
\hline Riesgo: & \multicolumn{2}{|c|}{ Alta } \\
\hline
\end{tabular}

c) Tarea de Definición del Diagrama de Clases (D-DC)

Por medio de esta tarea se implementa la segunda técnica que debe llevar a cabo el IR en la frase de Análisis Orientado al Producto, denominada Técnica de Creación del Diagrama de Clases (TC-DC) [13]. Para la aplicación de la TC-DC el IR dispone como producto de entrada Conceptos, Atributos, Valores, Tareas y Subtareas que fueron obtenidos a partir de la aplicación de las técnicas de la fase Análisis Orientado al Problema. Estos productos se procesan con la idea de identificar en los mismos, diferentes tipos de Clases, Objetos, Métodos y Relaciones que serán representados en el Diagrama de Clases (DC). Para comenzar a aplicar la (TCDC) el IR comienza por la identificación de Clases, Objetos y Métodos para luego generar las Relaciones en el 
DC como producto de salida que proporciona la (TC-DC). La tabla XXV resume los pasos y procedimientos necesarios para la implementación de esta técnica.

\section{TABLA XXIV. ESCENARIO DE CASO DE USO “ALQUILER DE} VIDEO"

\begin{tabular}{|c|c|}
\hline Nombre del Caso de Uso: & Alquiler de video \\
\hline Área: & Sistema de Tienda de Video \\
\hline Actor: & Empleado \\
\hline Descripción: & $\begin{array}{l}\text {... "Para un alquiler, el empleado debe registrar la fecha de } \\
\text { alquiler, dia de devolución, cliente, y número de cinta" ... }\end{array}$ \\
\hline Activar evento: & $\begin{array}{l}\text { E1 empleado, una vez que ingresa al módulo de "Alquiler", } \\
\text { carga los datos correspondientes y luego presiona el botón } \\
\text { "Ejecutar". }\end{array}$ \\
\hline Tipo de señal: & Externa \\
\hline Pasos desempeíados & Información para los pasos \\
\hline 1) Alquilar video & $\begin{array}{l}\text { Ingresa al módulo de "Alquilar", y carga los datos en los } \\
\text { campos obligatorios correspondientes a: cinta, cliente, } \\
\text { fecha de devolución y fecha de alquiler. }\end{array}$ \\
\hline 2) Ejecutar confirmación & $\begin{array}{l}\text { Luego, el empleado presiona el botón "Ejecutar" para } \\
\text { confirmar la operación. }\end{array}$ \\
\hline Precondiciones: & $\begin{array}{l}\text { E1 empleado realiza la identificación positiva para acceder a } \\
\text { las funcionalidades del software. }\end{array}$ \\
\hline Poscondiciones: & E1 empleado registra el alquiler de un video. \\
\hline Suposiciones: & $\begin{array}{l}\text { E1 empleado tiene un usuario, contraseña y el sistema instalado } \\
\text { en el ordenador. }\end{array}$ \\
\hline Reunir requerimientos: & $\begin{array}{l}\text { Esta acción permite dar registrar el alquiler de un video en el } \\
\text { sistema. }\end{array}$ \\
\hline Aspectos sobresalientes: & Generar un log de las transacciones efectuadas por el usuario. \\
\hline Prioridad: & Alta \\
\hline Riesgo: & Alta \\
\hline
\end{tabular}

TABLA XXV. TÉCNICA DE CREACIÓN DEL DIAGRAMA DE CLASES CON SUS ENTRADAS, SALIDAS Y PASOS

\begin{tabular}{|l|}
\hline \multicolumn{1}{|c|}{ Técnica de Creación del Diagrama de Clases (TC-DC) } \\
\hline Entrada: Tabla Concepto Atributo Valor (CAV) \\
Diagrama Jerárquico de Tareas (DJT) \\
Salidas: Diagrama de Clases (DC). \\
\hline Paso 1: Identificación de Clases, Objetos y Métodos. \\
Paso 2: Generar las Relaciones en el Diagrama de Clases (DC). \\
\hline
\end{tabular}

En los párrafos siguientes se describen los pasos de la técnica.

En este primer paso se realiza la identificación de Clases, Objetos, Métodos y Relaciones. Primero para identificar las Clases que constituyen el modelado, debemos extraer todos los Conceptos de la tabla CAV. Los Atributos del DC son los Atributos y Valores de los Conceptos de la tabla CAV. Cabe mencionar que en los casos que los Valores sean rangos de números o palabras determinadas, debemos resumirlo en el tipo de dato que es. Cuando se dé el caso de que los Valores sean "SI" o "NO", optaremos por elegir tipo de dato booleano. Por otra parte, los métodos de las clases son todas las Tareas del DJT.

En este segundo paso se explica las Relaciones entre Clases del diagrama. Como sabemos, la agregación es un tipo de vinculación que indica que una clase es parte de otra clase (composición débil). La destrucción del compuesto no conlleva a la destrucción de los componentes. La composición es una forma fuerte de asociación y la supresión del objeto compuesto conlleva la supresión de los componentes. La relación de Herencia indica que una clase (clase derivada) hereda los métodos y atributos especificados por una clase (clase base), por lo cual una clase derivada además de tener sus propios métodos y atributos, podrá acceder a las características y atributos visibles de su clase base. La relación entre clases conocida como Asociación, permite relacionar objetos que colaboran entre sí. Cabe destacar que no es una relación fuerte, es decir, el tiempo de vida de un objeto no depende del otro. Por ejemplo, se puede decir que una persona puede usar diferentes tipos de ropa, en cambio una ropa solo puede ser usada por una persona sola en un momento determinado. Para establecer las relaciones entre Clases, comenzaremos seleccionando una de ellas para compararla con el resto. Como primer paso debemos responder la siguiente pregunta: ¿Poseen atributos y/o métodos en común? Si esto es así, las clases deberán estar unidas por una línea recta. En caso contrario, las clases no estarán unidas y por lo tanto, pasaremos a la siguiente. Como paso posterior, debemos responder lo siguiente: ¿Puede existir esta clase sin la otra? Si la respuesta es no, estamos ante un caso de relación estructural de composición y será graficada con un rombo negro. Si los objetos se unen para lograr un fin en común pero a su vez son independientes entre sí, estamos ante un caso de relación estructural de asociación y será graficada mediante una línea, donde la punta de flecha indica el sentido de la asociación. En caso contrario, si las clases tienen una composición débil y una es parte de la otra clase, estamos ante una relación estructural de agregación, y será graficada con un rombo blanco. Al finalizar el modelado, si se observa que dos o más clases poseen relación con una clase y las mismas poseen atributos y/o métodos en común, la relación se deberá graficar con un triángulo blanco desde la clase base, uniendo las clases derivadas. Un ejemplo del subproducto que se obtiene se puede visualizar en la Figura 14.

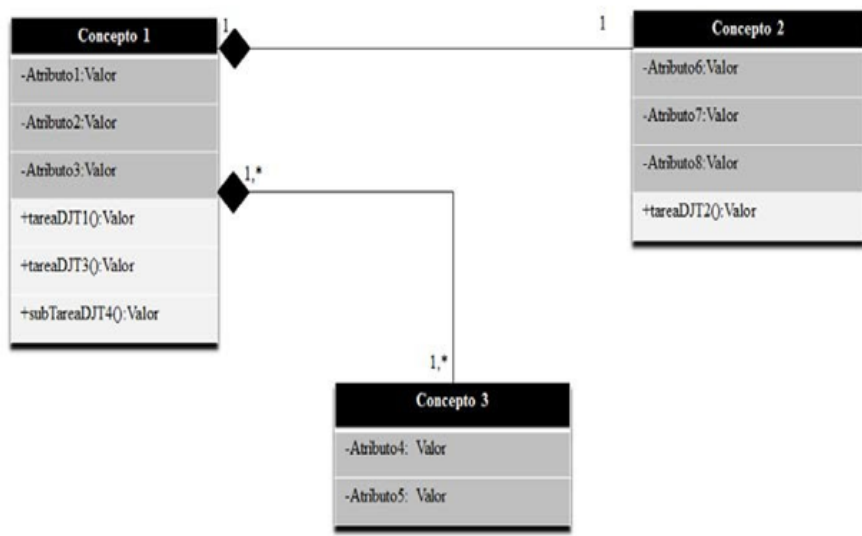

Fig. 14. Diagrama de Clases a partir de la técnica TC-DC

Como resultado de aplicar la técnica definida al caso de estudio propuesto, se obtienen la figura 15. En dicha figura se observan las clases, atributos, métodos y relaciones modelados a partir de los conceptos, atributos y valores de la tabla CAV. Los métodos que se observan son identificados a partir del DJT como se mencionó anteriormente.

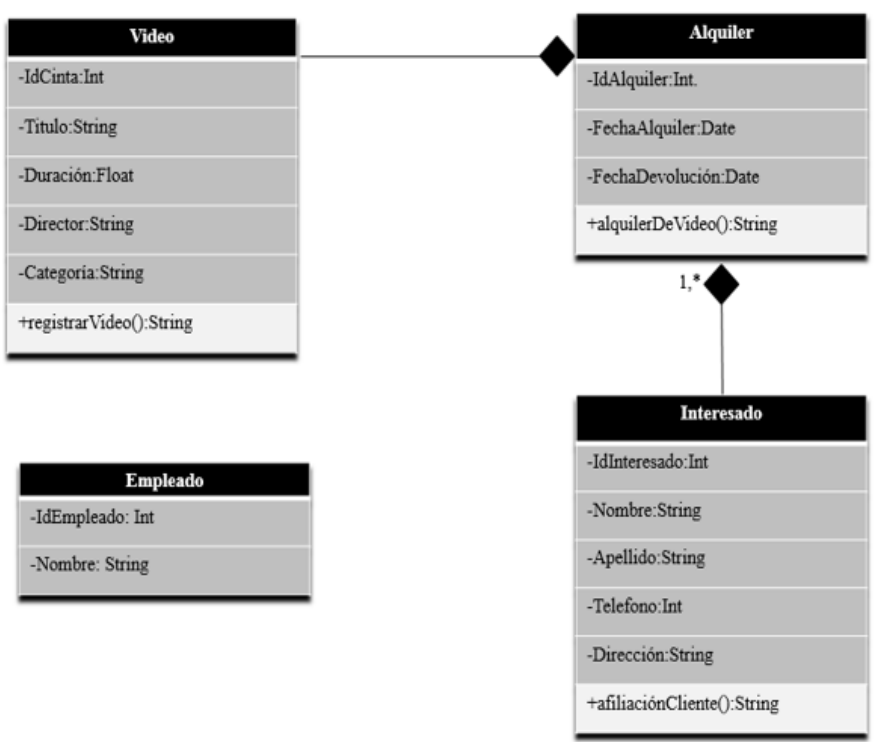

Fig. 15. Diagrama de Clases Tienda de Video. 


\section{Modelos no alcanzados por la propuesta}

Esta sección hace mención a los modelados que no son aplicables a esta Propuesta de Conceptualización de Requisitos para Proyectos Software basados en formalismos de Ingeniería de Conocimiento. Por una dependencia conceptual y lógica de conceptos generados en la segunda fase denominada "Análisis Orientado al Producto", los Diagramas de Secuencia y los Diagramas de Entidad Relación no surgen a partir de esta propuesta, sino que son originados a partir de las técnicas convencionales.

\section{Resumen de la propuesta}

En esta sección se resume todas las representaciones generadas a partir de la aplicación de la Propuesta. En la figura 16 se ilustra el flujo de pasos que conlleva la ejecución del Proceso de Conceptualización de Requisitos, las interdependencias entre las fases, sus tareas y sus productos, representadas mediante el ingreso como elemento de entrada de un paso, uno o más elementos de salida de otro. En dicha figura se presentan las ocho tareas de la propuesta, distribuidas según las fases a las que pertenecen.

Dichas tareas se conectan mediante líneas y flechas, las cuales indican los elementos de entrada (aquellas que convergen con la parte izquierda del paso) y salidas (aquellas que surgen del sector derecho del paso) correspondientes a cada tarea y como cada elemento de salida retroalimenta otra tarea posterior. El orden de ejecución de los pasos se define a continuación, identificando las fases con negrita, las tareas encasilladas en rectángulos, y los productos de entrada/salida con su imagen en miniatura: La Técnica de Segmentación del Discurso del Usuario (TS-DU) genera como producto de salida la Tabla de Segmentos de Texto (T-ST) a partir del Discurso de Usuario (DU) como producto de entrada. La Técnica Cognitiva de Identificación de Conceptos Atributos $y$ Valores (TC-CAV) genera como producto de salida la Tabla Concepto Atributo Valor (CAV) a partir de las Tablas de Segmentos de Texto (T-ST). La Técnica Cognitiva de Identificación de Acciones, Condiciones y Vínculos (TCACV) genera como producto de salida las Tablas Palabras Experto Regla Vínculo (PERV) a partir de las Tablas de
Segmento de Texto (T- ST). La Técnica de Confección del Diccionario (TC-D) genera como producto de salida el Diccionario (D) a partir de las Tablas de Segmento de Texto (T-ST) y la Tabla Concepto Atributo Valor (CAV). La Técnica de Confección del Diagrama Jerárquico de Tareas (TC-DJT) genera como producto de salida el Diagrama Jerárquico de Tareas (DJT) a partir de las Tablas Palabras Experto Regla Vínculo (PERV) y la Tabla Concepto Atributo Valor (CAV). La Técnica de Armado del Diagrama de Casos de Uso (TA-DCU) genera como producto de salida el Diagrama de Casos de Uso (DCU) a partir de las Tablas CAV y los Diagramas Jerárquicos de Tareas (DJT). La Técnica de Creación de Escenarios de Casos de Uso (TC-ECU) genera como producto de salida los Escenarios de Caso de Uso (ECU) a partir de las Tablas CAV, PERV, Diagramas de Casos de Uso (DCU) y Diagrama Jerárquico de Tareas (DJT). Por último, la Técnica de Creación del Diagrama de Clases (TC-DC) genera como producto de salida el Diagrama de Clases (DC) a partir de la Tabla CAV y el Diagrama Jerárquico de Tareas (DJT). Finalmente, como resultado del proceso, se obtiene como objetivo la conceptualización de requisitos para el sistema software en cuestión.

\section{CONCLUSIONES}

En esta sección se presentan las aportes de este artículo (sección V.A) y se destacan las futuras líneas de investigación que se consideran de interés en base al problema abierto presentado (sección V.B).

\section{A. Aportes del artículo}

En este artículo se ha definido un proceso que permite, partiendo del discurso del usuario, minimizar la brecha conceptual para alcanzar la representación de requisitos. Además se han propuesto un proceso y una serie formalismos de representación de conocimiento que permiten documentar la información necesaria para llevar a cabo la ejecución de la Propuesta de Conceptualización de Requisitos para Proyectos Software basados en formalismos de Ingeniería de Conocimiento.

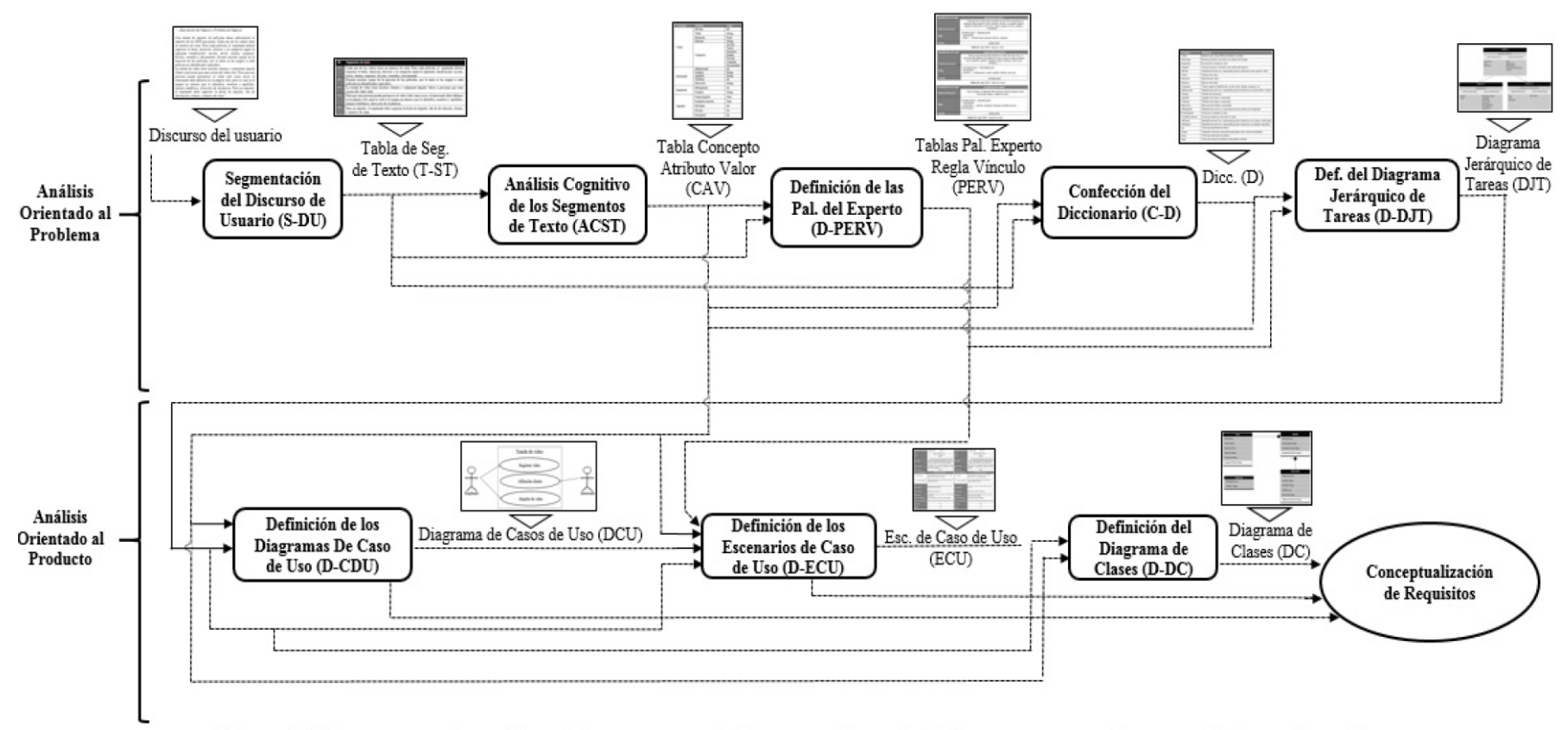

Fig. 16. Proceso de Conceptualización de Requisitos Basado en Ingenieria del Conocimiento 
En este contexto, este artículo ha propuesto:

I. Una propuesta de conceptualización de requisitos para proyectos software basados en formalismos de IC que se desarrolla en dos fases: orientada al análisis del problema y orientada al Análisis del producto.

II. Para la Fase de Análisis Orientado al Problema se han propuesto las siguientes tareas: [i] Segmentación del Discurso de Usuario, la cual necesita del Discurso de Usuario como producto de entrada y proporciona como producto de salida los correspondientes Segmentos de Texto, [ii] Análisis Cognitivo de los Segmentos de Texto, que toma como producto de entrada a los Segmentos de Texto y proporciona como producto de salida la correspondiente Tabla Concepto Atributo Valor $(C A V)$, [iii] Definición de las Palabras del Experto, que toma como producto de entrada a los Segmentos de Texto y proporciona como producto de salida las correspondientes Tablas Palabras Experto Regla Vínculo (PERV), [iv] Confección del Diccionario que toma como producto de entrada los Segmentos de Texto y la Tabla CAV y proporciona como producto de salida el Diccionario, [v] Definición del Diagrama Jerárquico de Tareas que toma como producto de entrada las Tablas PERV y la Tabla $C A V$, y proporciona como producto de salida el Diagrama Jerárquico de Tareas (DJT).

III. Para la Fase de Análisis Orientado al Producto, se ha propuesto las siguientes tareas: [vi] Definición de los Diagramas de Caso de Uso, la cual necesita como productos de entrada a la Tabla $C A V$ y el Diagrama Jerárquico de Tareas, los cuales se procesan en el desarrollo.

La propuesta de conceptualización de requisitos para proyectos software basados en formalismos de IC, las etapas y técnicas asociadas han sido validadas en tres dominios de conocimiento con características bien diferenciadas: el primero sobre una empresa de prestación de servicios de hotelería, el segundo correspondiente a la gestión de tickets para reparación de Hardware y el tercero correspondiente a una entidad emisora de tarjetas de crédito y débito.

\section{B. Futuras líneas de investigación}

Durante el desarrollo de este proyecto de artículo han surgido cuestiones que si bien no son centrales al tema abordado en la misma, constituyen temas concomitantes que (en opinión del autor) darían lugar a las siguientes futuras líneas de investigación:

- La validación empírica más amplia del proceso propuesto mediante la técnica de muestras apareadas basadas en grupos experimental y de control.

- La validación empírica del proceso y de las técnicas propuestas en un conjunto vasto y representativo, considerando las distintas complejidades de dominios y problemas del negocio.

- Implementar estos formalismos en las cursadas de Ingeniería de Software I para verificar y validar su correcto funcionamiento con mayor amplitud que con los casos de validación propuestos en este artículo.

\section{REFERENCIAS}

[1] Pytel, P., Uhalde, C., Ramón, H. D., Castello, H., Tomasello, M., Pollo Cattaneo, M. F. \& García Martínez, R. (2011). Ingeniería de requisitos basada en técnicas de ingeniería del conocimiento. In XIII Workshop de Investigadores en Ciencias de la Computación.

[2] Hossian, A. (2012). Modelo de proceso de conceptualización de requisitos (Doctoral dissertation, Facultad de Informática).

[3] Rumbaugh, J., Booch, G., \& Jacobson, I. (2000). El lenguaje unificado de modelado: manual de referencia.

[4] Britos, 2008. Modelados de Conocimiento. Ingeniería de Software.

[5] Davis, A. 1993. Software Requirements: Objects, Functions and States; Prentice-Hall International.

[6] Faulk, S. 1997. Software Requirements: A Tutorial; In Software Engineering, IEEE Computer Society Press, pp 82101.

[7] Kaindl, H. 1999. Difficulties in the transition from OO analysis to design. IEEE Software, 16(5).

[8] Sutcliffe, A., Maiden, N. 1992. Analysing the Novice Analyst: Cognitive Models in Software Engineering; International Journal of Man-Machine Studies, 36(5).

[9] Robertson, S., Robertson, J. (1999). Mastering the Requirements Process. Addison-Wesley.

[10] Sánchez, J. P. (2012). Propuesta de Estandarización de Procesos (Doctoral dissertation, Tesis de Maestro en Administración).

[11] Gómez, A., N. Juristo, C. Montes, J. Pazos, Ingeniería del Conocimiento, Ed. Centro de Estudios. Ramón Areces, (1997).

[12] García Martínez, R. y Britos, P. 2004. Ingeniería de Sistemas Expertos. Editorial Nueva Librería. ISBN 987-1104-15-4.

[13] Steeven, P., Pooley, R., Alarcón, M. F., \& Crespo, R. G. (2007). Utilización de UML en Ingeniería del Software con Objetos y Componentes.

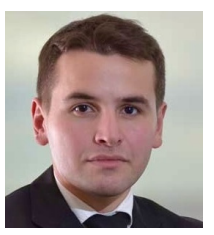

Lautaro Ignacio Ferrer. Es Asistente de Investigación del Laboratorio de Ingeniería del Software. Su investigación se centra en "Conceptualización de requisitos para Proyectos Software basados en formalismos de Ingeniería de Conocimiento" con radicación como linea de trabajo del proyecto UNLa 33B170 "Formulación de un Modelo de Proceso para Ingeniería de Conocimiento" perteneciente al Grupo de Investigación en Sistemas de Información (UNLa GISI) del Departamento de Desarrollo Productivo y Tecnológico de la Universidad Nacional de Lanús.. 